\title{
Identification of IncRNAs Responsive to Infection by Plasmodiophora brassicae in Clubroot-Susceptible and -Resistant Brassica napus Lines Carrying Resistance Introgressed from Rutabaga
}

\author{
Aarohi Summanwar, Urmila Basu, Habibur Rahman, ${ }^{\dagger}$ and Nat $\mathrm{Kav}^{\dagger}$ \\ Department of Agricultural, Food and Nutritional Science, University of Alberta, 4-10 Agriculture/Forestry Centre, Edmonton, \\ AB, T6G 2P5, Canada
}

Accepted 13 May 2019.

\begin{abstract}
Clubroot disease, caused by Plasmodiophora brassicae Woronin, is a major threat to the production of Brassica' crops. Resistance to different $P$. brassicae pathotypes has been reported in the $A$ genome, chromosome A08; however, the molecular mechanism of this resistance, especially the involvement of long noncoding RNAs (IncRNAs), is not understood. We have used a strandspecific IncRNA-Seq approach to catalog IncRNAs from the roots of clubroot-susceptible and -resistant Brassica napus lines. In total, 530 differentially expressed (DE) IncRNAs were identified, including $88 \%$ of long intergenic RNAs and $11 \%$ natural antisense transcripts. Sixteen IncRNAs were identified as target mimics of the microRNAs (miRNAs) and eight were identified as the precursors of miRNAs. KEGG pathway analysis of the DE IncRNAs showed that the cis-regulated target genes mostly belong to the phenylpropanoid biosynthetic pathway (15\%) and plant-pathogen interactions $(\mathbf{1 5 \%})$ while the transregulated target genes mostly belong to carbon $(18 \%)$ and amino acid biosynthesis pathway (19\%). In all, 24 DE IncRNAs were identified from chromosome A08, which is known to harbor a quantitative trait locus conferring resistance to different $P$. brassicae pathotypes; however, eight of these IncRNAs showed expression only in the resistant plants. These results could form the basis for future studies aimed at delineating the roles of IncRNAs in plant-microbe interactions.
\end{abstract}

Keywords: canola, clubroot, genomics, long non-coding RNAs, metabolomics, Plasmodiophora brassicae, plant responses to pathogens, primary metabolism, proteomics, RNA-seq

Intergenic regions in the genome have been shown to be involved in transcriptional and posttranscription regulation of

${ }^{\dagger}$ Corresponding authors: N. Kav; nat@ualberta.ca; and H. Rahman; Habibur.rahman@ualberta.ca

Funding: We thank the Alberta Crop Industry Development Fund (ACIDF), the Alberta Canola Producers Commission (ACPC), Agriculture and Agri-Food Canada, and the Natural Sciences and Engineering Research Council of Canada for financial support of this project.

*The $\boldsymbol{e}$-Xtra logo stands for "electronic extra" and indicates that three supplementary figures and 11 supplementary tables are published online.

The author(s) declare no conflict of interest.

๑) 2019 The American Phytopathological Society gene expression (Kapranov et al. 2007). These regions are ubiquitously transcribed into RNAs and are classified based on their different sizes and their regulatory functions. These types of functional RNAs are classified as noncoding RNAs (ncRNAs) (Ariel et al. 2015; Kapranov et al. 2007; Wilusz et al. 2009). Long ncRNA (lncRNAs) are defined to be greater than 200 bp (Chen 2009; Kapranov et al. 2007) and have been implicated in various gene regulatory processes, including the reprogramming of gene expression in plants in response to a variety of biotic and abiotic stresses (Nejat and Mantri 2018).

lncRNAs have been classified into different categories based on their genomic location and orientation. These include promoter upstream transcripts, enhancer RNAs, intergenic ncRNAs (lincRNAs), intronic ncRNAs, bidirectional lncRNAs, overlapping lncRNAs, and natural antisense transcripts (NATs) (Mattick and Rinn 2015; Morris and Mattick 2014; Wu et al. 2017). Similar to the mRNA transcription process, lncRNAs are also transcribed by RNA polymerase II in plants and are modified with a $5^{\prime}$-cap and $3^{\prime}$-end poly-adenylation (Guttman et al. 2009).

Advances in the next-generation sequencing have enabled genomewide identification of lncRNAs in many eukaryotes, including several plant species. The first systematic investigation on plant lncRNAs began with the identification of 6,480 lincRNAs in Arabidopsis thaliana through bioinformatics analysis; however, expression profiling using microarray and RNA-seq confirmed 2,708 transcripts to be lincRNAs (Liu et al. 2012). Similarly, exploitation of expressed sequence tag databases and 30 different RNA-seq datasets resulted in the identification of 20,163 putative lncRNAs (Li et al. 2014). Among these, 1,704 were considered to be high-confidence lncRNAs in maize, which did not exhibit sequence similarity with known ncRNAs. Most ( $>90 \%$ ) of the lncRNAs were found to be in the intergenic regions, with more than $50 \%$ of the lncRNAs observed to be expressed in a tissue-specific manner and largely affected by transgenetic factors (Li et al. 2014).

Regulatory roles for the lncRNAs have also been reported in many plant species; for example, in cellulose, lignin, and gibberellin biosynthesis as well as in wood formation in Populus tomentosa (Chen et al. 2015). Such regulatory roles have also been observed in flower and fruit development in diploid strawberry Fragaria vesca (C. Y. Kang and Liu 2015) and trifoliate orange Poncirus trifoliata L. Raf. (Wang et al. 2017). In Brassica campestris, Song et al. (2013) identified an lncRNA, BcM11, involved in pollen development. Additional studies have supported a 
crucial role of lncRNAs in plant growth and development, disease response, and genetic or epigenetic regulation of traits (Huang and Zhang 2014; Huang et al. 2018; Nejat and Mantri 2018).

lncRNAs are also known as the class of riboregulators that are expressed in response to plant biotic stress. Zhu et al. (2014) identified 35 lncRNAs, including 15 NATs and 20 lincRNAs, involved in resistance to Fusarium oxysporum in A. thaliana. Of these, antisense transcription in approximately $20 \%$ of the annotated $A$. thaliana genes was observed. The role of lncRNAs in response to diseases has also been documented in many other crop species; for example in tomato against Tomato yellow leaf curl virus (Wang et al. 2015); in B. napus in response to Sclerotinia sclerotiorum, which causes stem rot disease (Joshi et al. 2016); as well as in the forest tree genus Populus in response to Melampsora larici-populina (N. Wang et al. 2017). It has also been reported that overexpression of the lncRNA16397 in tomato induces the expression of glutaredoxin, reduces the accumulation of reactive oxygen species, and, thus, confers resistance to Phytophthora infestans (Cui et al. 2017). Recently, L. Zhang et al. (2018) reported that silencing of lncRNAs GhlncNAT-ANX2 and GhlncNAT-RLP7, which are involved in the regulation of lipoxygenase activity, results in enhanced resistance toward Verticillium dahliae and Botrytis cinerea. ELF18-induced long noncoding RNA 1 is another functionally characterized lncRNA, which enhances resistance against Pseudomonas syringae in A. thaliana by positively regulating pathogenesis-related $1(P R I)$ gene expression (Seo et al. 2017). Taken together, all of these reports suggest a significant role for lncRNAs in response and resistance to diseases in plants and warrant continued investigation. To our knowledge, no study has been conducted thus far to understand the role of lncRNAs in resistance to other diseases such as the clubroot (CR) disease of Brassica crops.

$\mathrm{CR}$ is a soilborne disease affecting cruciferous, crops including canola, cabbage, turnip, Brussels sprout, and many other Brassica crops (Howard et al. 2010). It is caused by a soilborne, obligate parasite, Plasmodiophora brassicae Woronin, a member of the eukaryotic subgroup Rhizaria (Burki et al. 2010). $P$. brassicae is a fungus-like protist which is known to possess a complex lifecycle and is unable to grow and multiply without a living host (Hwang et al. 2012). This pathogen can stay in soil for over 15 years (Wallenhammar 1996) in the form of resting spores and germinates only in the presence of secretions from the plant roots (Macfarlane 1970). Disease development leads to the formation of galls on the roots, which negatively affect water and nutrient uptake by roots, leading to stunted growth (Dixon 2009). In the case of the most severely affected fields, CR disease can cause yield loss of approximately $30 \%$ in canola (Tewari et al. 2005). Due to the nature of the occurrence of this disease and its perpetual infestation cycle, it is difficult to control this disease with just implementation of improved agricultural practices and chemical treatments (Dobson et al. 1983; Howard et al. 2010; Kowata-Dresch and May-De Mio 2012).

Despite the existence of commercial, CR-resistant cultivars and integrated management approaches, it is still crucial to achieve a better understanding of plant responses to $P$. brassicae infection and to develop cultivars with durable resistance, in order to combat genetic shifts in this pathogen population and subsequent loss of effective resistance (Peng et al. 2014). Genome and proteome analyses of various plant species of the family Brassicaceae challenged with $P$. brassicae have provided some insights about molecular mechanisms triggering resistance in the host plants (Cao et al. 2008; Chen et al. 2016; Devos et al. 2006; Zhang et al. 2016). The availability of the Brassica napus genome sequence (Chalhoub et al. 2014) has now paved the way for investigation into the ncRNA and their regulatory roles in Brassica spp. The objective of this research was to identify the lncRNAs involved in the regulation of resistance to $P$. brassicae by using B. napus canola lines carrying resistance introgressed from rutabaga (Hasan and Rahman 2016).

\section{RESULTS}

\section{Microscopic examination}

of $P$. brassicae infection of $B$. napus.

Microscopic examination of the roots of the resistant $(\mathrm{R})$ and susceptible $(\mathrm{S})$ plants inoculated with $P$. brassica pathotype 3 showed no evidence of infection at 6 days postinoculation (dpi) (Table 1). Infection was observed in the secondary roots at 10 dpi in both S (Fig. 1C) and R (Fig. 1D) plants. Approximately $7.3 \%$ of cells were observed to be infected in $S$ while $2.3 \%$ of cells were observed in $\mathrm{R}$ (Table 1 ); however, there was no swelling of the roots in either S or R. At $14 \mathrm{dpi}, 8.3 \%$ cells were observed to be infected in S compared with $2.7 \%$ in $\mathrm{R}$ (Table 1). A greater number of primary plasmodia were observed in the $S$ plants as compared with the R plants (data not shown). At 18 dpi (Fig. 1E and F), approximately $51.7 \%$ of cells showed the presence of secondary plasmodium in S plants whereas $2.7 \%$ of cells in $\mathrm{R}$ already showed the presence of resting spores. The root cells of the S plants started to show evidence of hypertrophy at 18 dpi (Fig. 1E). As expected, there was no indication of gall formation in the inoculated $\mathrm{R}$ plants and they were morphologically similar to the uninoculated R and S plants. At 22 dpi (Fig. $1 \mathrm{G}$ and $\mathrm{H}$ ), more than $60 \%$ of cortical cells of the $S$ plants showed the presence of mature secondary plasmodia and resting spores (Table 1), whereas $2.7 \%$ of infected cortical cells with resting spores could be seen in the R plants, because there was no further increase in infection observed. In conclusion, this study showed that infection by $P$. brassicae can occur in both $\mathrm{S}$ and $\mathrm{R}$ plants at $10 \mathrm{dpi}$; however, the infection did not progress further in the $\mathrm{R}$ plants. Based on these results, we focused on the root samples collected at 10 dpi for studies on lncRNA profiling.

\section{Identification of IncRNAs \\ in $B$. napus following $P$. brassicae infection.}

Strand-specific lncRNA-Seq libraries were prepared from the roots of the $\mathrm{R}$ and $\mathrm{S}$ plants collected at 10 dpi to identify the IncRNAs responsive to $P$. brassicae infection. In total, 461 million paired-end clean reads were obtained from six strandspecific RNA-seq libraries (Table 2). The error rate was $0.01 \%$, with Q30 percentage exceeding 98\% for all the six libraries. After removal of the low-quality sequences and the adapter sequences and artifacts, 367 million $(79.8 \%)$ clean reads were mapped on to the reference $B$. napus genome; however, by use of TopHat (v2.0.9) (Trapnell et al. 2012), 73.5\% of the reads could be uniquely mapped, followed by assembly and annotation of all the transcripts in B. napus using Cufflinks (v2.1.1) (Trapnell et al. 2010). From the total 198,553 assembled transcripts, $78.0 \%$ of transcripts were found to be mRNAs after the application of exon number and transcript length filters, which

Table 1. Percentage of infected cells observed in clubroot-susceptible (S) and -resistant (R) Brassica napus lines as observed microscopically at different time points ${ }^{\mathrm{a}}$

\begin{tabular}{lcc}
\hline Time point & S lines & R lines \\
\hline 0 hpi & 0 & 0 \\
6 dpi & 0.7 & 0 \\
$10 \mathrm{dpi}$ & 7.3 & 2.3 \\
$14 \mathrm{dpi}$ & 8.3 & 2.7 \\
$18 \mathrm{dpi}$ & 51.7 & 2.7 \\
$22 \mathrm{dpi}$ & 66.0 & 2.7 \\
\hline
\end{tabular}

${ }^{\mathrm{a}}$ hpi $=$ hours postinoculation and dpi $=$ days postinoculation; 300 cells were examined microscopically for each time point. 


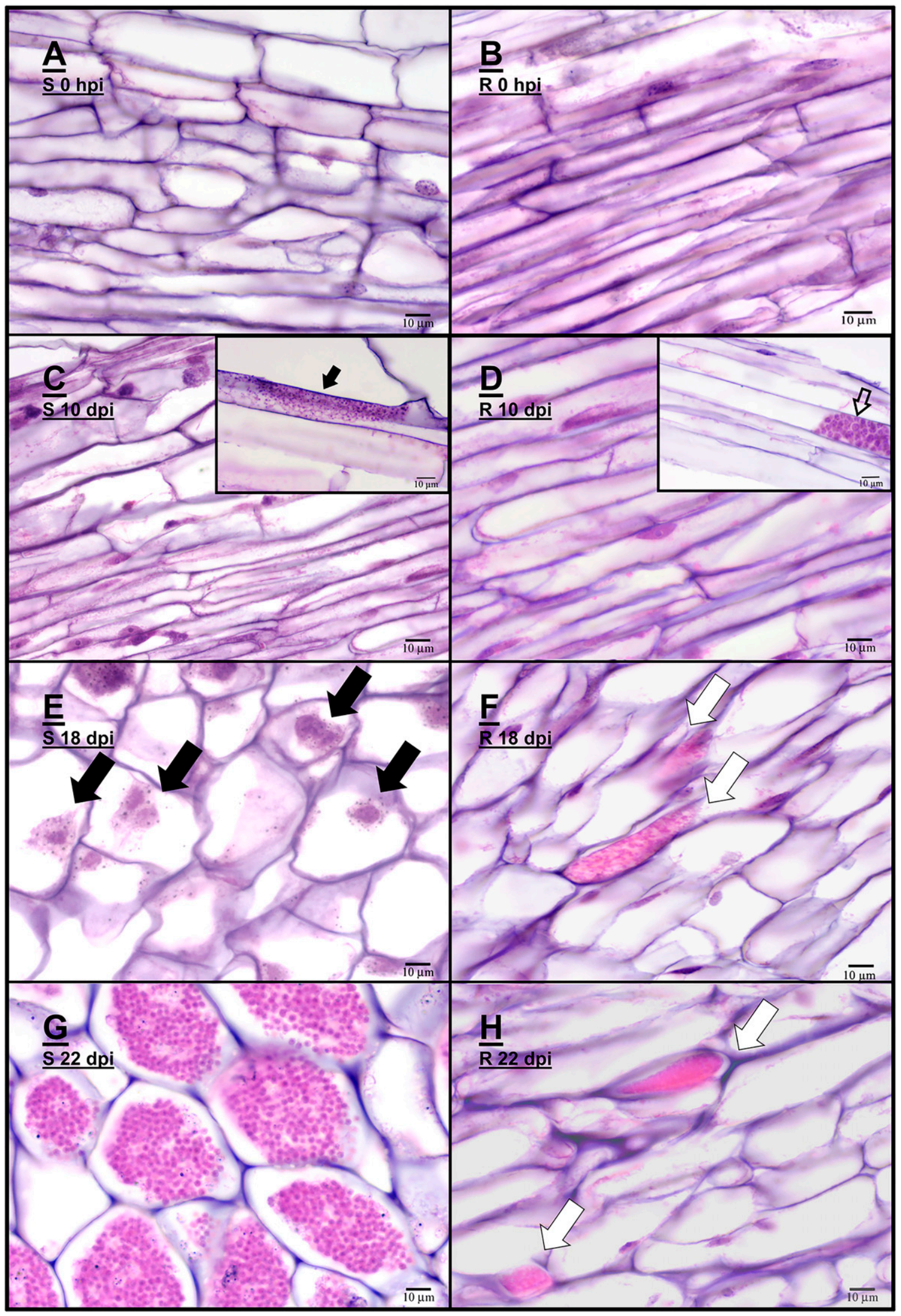

Fig. 1. Different stages of Plasmodiophora brassicae infection observed in histopathological analysis of Brassica napus roots at $\mathbf{A}$ and $\mathbf{B}, 0 \mathrm{~h}$ postinoculation (hpi) and 10, 18, and 22 days postinoculation (dpi). C and D, Presence of proliferating zoosporangia in both rutabaga-derived clubroot-susceptible (S) and clubroot-resistant (R) Brassica napus at 10 dpi (inset shows larger magnification); $\mathbf{E}$ and $\mathbf{F}$, matured secondary plasmodia in the cortical cells of $\mathbf{S}$ (black arrows) and resting spores in few cortical cells of $\mathrm{R}$ (white arrows) at $18 \mathrm{dpi}$; and $\mathbf{G}$ and $\mathbf{H}$, many resting spores and secondary plasmodia in $\mathrm{S}$ and resting spores in very few cortical cells of $\mathrm{R}$ (white arrows) at $22 \mathrm{dpi}$. Bars represent the resolution of the microscopy for the particular section. 
were further filtered to remove the transcripts showing an overlap with the exon regions of the annotated functional genes (as identified using Cuffcompare, v2.1.1) (Trapnell et al. 2010). Expression of these transcripts was calculated using Cuffdiff (v2.1.1) and the transcripts with fragments per kilobase of transcript per million mapped reads $(\mathrm{FPKM}) \geq 0.5$ were selected for further analysis. The Coding Potential Calculator (CPC) (Kong et al. 2007) and Pfam Scan (v1.3) (Punta et al. 2012) were used to filter the remaining transcripts for their coding potential to distinguish the protein-coding and noncoding sequences and, in total, 4,558 transcripts were deemed to be lncRNAs. These lncRNAs included $84.7 \%$ longintergenic RNAs and $15.3 \%$ antisense RNAs. Screening of the putative lncRNAs based on $q$ value $(<0.05)$ identified 530 differentially expressed (DE) lncRNAs between the R and S plants, and this included $58(10.9 \%)$ antisense lncRNAs and $472(89.1 \%)$ lincRNAs.

\section{Characterization and functional analysis of DE IncRNAs in response to $P$. brassicae infection.}

More than $60 \%$ of the lncRNAs were observed to be 200 to 1,000 nucleotides (nt) in size and the lengths of the $530 \mathrm{DE}$ lncRNAs ranged from 206 to 8,584 nt. Approximately 18\% (96 of 530) lncRNAs were longer than $1.5 \mathrm{~kb}$ nt in size (Supplementary Fig. S1). Analysis of all of the DE lncRNAs for the size of their exons indicated that $78 \%$ (663 of 853 exons) had a length of 18 to $1,000 \mathrm{nt}$. Moreover, lncRNAs were observed to be significantly shorter compared with mRNAs. The number of transcripts for lncRNAs with a single exon constituted approximately $62 \%$ (329 of 530) of these lncRNAs, while $26 \%$ (138 of 530) consisted of two exons and only $2.6 \%$ (14 of 530) lncRNAs contained more than five exons. The DE lncRNAs were evenly distributed across the $19 \mathrm{~B}$. napus chromosomes. Of the total 530 DE lncRNAs, 184 and 229 were observed to be located on the 10 "A" chromosomes and the nine " $\mathrm{C}$ " chromosomes, respectively, of the B. napus genome. However, 117 DE IncRNAs could not be assigned to any specific chromosome. A strong correlation (0.76) was observed between the distribution of the number of DE lncRNAs and chromosome size. When the distribution of DE lncRNAs on individual chromosomes was considered, the highest number was found on chromosome $\mathrm{C} 03$, followed by chromosomes $\mathrm{C} 09$, $\mathrm{A} 09$, and A03. Interestingly, chromosome A08, to which the CR resistance gene has been previously mapped, was found to harbor 24 DE lncRNAs.

Of the 530 DE lncRNAs, 345 were upregulated and 185 were downregulated in the $\mathrm{S}$ plants as compared with $\mathrm{R}$ plants (Supplementary Fig. S2). In contrast, 170 lncRNAs were upregulated and 337 were downregulated in the R plants as compared with S plants. In all, 15 lncRNAs were detected only in the R plants while eight lncRNAs were detected only in the $S$ plants (Table 3). The top 20 upregulated and downregulated

Table 2. Mapping statistics of the sequencing reads from six-stranded Brassica napus libraries postinfection with Plasmodiophora brassicae

\begin{tabular}{lcrrr}
\hline & \multicolumn{4}{c}{ Number of reads (\%) } \\
\cline { 2 - 5 } Replicates $^{\mathbf{a}}$ & \multicolumn{1}{c}{ Total } & Total mapped & Multiple mapped & Unique mapped \\
\hline R_1 & $78,145,602$ & $62,866,707(80.45)$ & $4,874,861(6.24)$ & $57,991,846(74.21)$ \\
R_2 & $77,724,002$ & $62,072,779(79.86)$ & $4,964,474(6.39)$ & $57,108,305(73.48)$ \\
R_3 & $72,187,808$ & $57,818,092(80.09)$ & $4,454,507(6.17)$ & $53,363,585(73.92)$ \\
S_1 & $85,702,896$ & $68,181,904(79.56)$ & $5,206,587(6.08)$ & $52,975,317(73.48)$ \\
S_2 & $72,543,352$ & $57,417,259(79.15)$ & $4,640,617(6.4)$ & $54,876,642(72.75)$ \\
S_3 & $74,959,046$ & $59,616,509(79.53)$ & $4,735,211(6.32)$ & $(73.22)$ \\
Total & $461,262,706$ & $367,973,250(79.77)$ & $28,876,257(6.26)$ & $339,096,993(73.51)$ \\
\hline
\end{tabular}

${ }^{a} \mathrm{R} \_1, \mathrm{R} \_2$, and $\mathrm{R} \_3$ are the biological replicates of clubroot $(\mathrm{CR})$-resistant $B$. napus and $\mathrm{S} \_1, \mathrm{~S} \_2$, and $\mathrm{S} \_3$ are the biological replicates of CR-susceptible B. napus.

Table 3. Long noncoding RNAs (lncRNAs) uniquely expressed in clubroot disease-resistant (R) lines carrying the resistance gene of rutabaga and clubrootsusceptible (S) lines of Brassica napus ${ }^{\mathrm{a}}$

\begin{tabular}{|c|c|c|c|}
\hline Transcript ID & Chromosome name and gene location & S (FPKM) & R (FPKM) \\
\hline LNC_001161 & A08: $12,183,521-12,183,982$ & $\mathbf{0}$ & 0.954916 \\
\hline LNC_001063 & A08: 3,202,637-3,204,852 & $\mathbf{0}$ & 1.02438 \\
\hline LNC_000600 & A04: $14,816,008-14,816,758$ & 0 & 1.3608 \\
\hline LNC_001126 & A08: 3,447,903-3,448,931 & $\mathbf{0}$ & 1.7121 \\
\hline LNC_000450 & A03: $27,099,999-27,101,445$ & 0 & 1.98082 \\
\hline LNC_002916 & C04: 47,030,126-47,030,803 & 0 & 1.98632 \\
\hline LNC_001146 & A08: 9,573,084-9,573,548 & $\mathbf{0}$ & 2.28077 \\
\hline LNC_000108 & A01: $19,635,847-19,636,338$ & 0 & 2.30396 \\
\hline LNC_001065 & A08: 3,788,461-3,789,000 & $\mathbf{0}$ & 2.92531 \\
\hline LNC_001154 & A08: $10,153,693-10,154,043$ & $\mathbf{0}$ & 3.7843 \\
\hline LNC_001089 & A08: $12,365,659-12,366,252$ & $\mathbf{0}$ & 5.01747 \\
\hline LNC_004045 & C09: 43,229,826-43,230,101 & 0 & 6.3523 \\
\hline LNC_001851 & Ann_random: 41,970,174-41,971,127 & 0 & 1.4904 \\
\hline LNC_001191 & A08_random: $1,215,048-1,217,686$ & $\mathbf{0}$ & 3.3501 \\
\hline LNC_001888 & Ann_random: $47,682,468-47,684,525$ & 0 & 5.2681 \\
\hline LNC_000089 & A01: $12,314,342-12,314,908$ & 2.07049 & 0 \\
\hline LNC_001341 & A09: $11,766,717-11,767,907$ & 2.1138 & 0 \\
\hline LNC_002432 & C03: 26,497,021-26,497,291 & 5.19373 & 0 \\
\hline LNC_003616 & C07: 28,269,085-28,273,791 & 1.93572 & 0 \\
\hline LNC_004790 & Unn_random: 8,133,944-8,134,628 & 0.8604 & 0 \\
\hline LNC_001807 & Ann_random: $35,009,658-35,010,033$ & 1.4708 & 0 \\
\hline LNC_000887 & A06_random: $299,855-300,207$ & 1.5085 & 0 \\
\hline LNC_000736 & A05_random: $1,032,000-1,032,588$ & 0.8626 & 0 \\
\hline
\end{tabular}

\footnotetext{
${ }^{\text {a }}$ FPKM $=$ fragments per kilobase of transcript per million mapped reads. Rows in bold indicate the lncRNAs located on chromosome A08.
} 
lncRNAs of the R plants are presented in Supplementary Table S2. In all, 4 of the top 20 downregulated lncRNAs in R are located on chromosome A08. Recent insights from functional analysis of the lncRNAs have demonstrated that they can be involved in regulating the expression of the overlapping or neighboring genes (cis-acting) or genes located at a long distance (transacting). Genomic regions $100 \mathrm{~kb}$ upstream and downstream of the IncRNAs were scanned to predict the colocated target genes of cis-acting lncRNAs (Supplementary
Table S3). All 530 DE lncRNAs were found to pair with the annotated mRNAs from both upstream and downstream regions (Supplementary Fig. S3). KEGG pathway enrichment analysis revealed that the majority of the colocated target genes were from plant-pathogen interactions $(15 \%)$ and the phenylpropanoid biosynthetic pathway (15\%) (Fig. 2A). Similarly, 425 DE lncRNAs were predicted to have coexpressed targets based on correlation of expression between the lncRNAs and mRNAs. KEGG pathway enrichment analysis also revealed

\section{A Pathway enrichment analysis for the co-located targets}

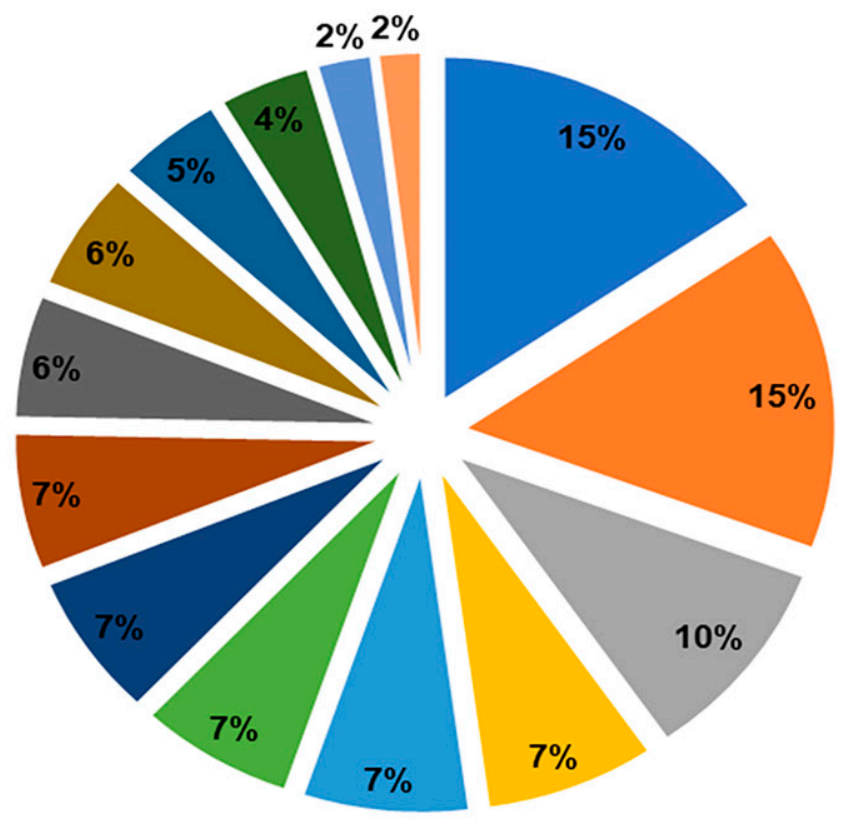

- Plant-pathogen interaction

- Phenylpropanoid biosynthesis

- Glycolysis / Gluconeogenesis

- Pyruvate metabolism

- Glyoxylate and dicarboxylate metabolism

- Phosphatidylinositol signaling system

- 2-Oxocarboxylic acid metabolism

- Glycerolipid metabolism

- Fructose and mannose metabolism

- Citrate cycle (TCA cycle)

- Basal transcription factors

- Steroid biosynthesis

- Sphingolipid metabolism

Biotin metabolism

\section{B Pathway enrichment analysis for the co-expressed targets}

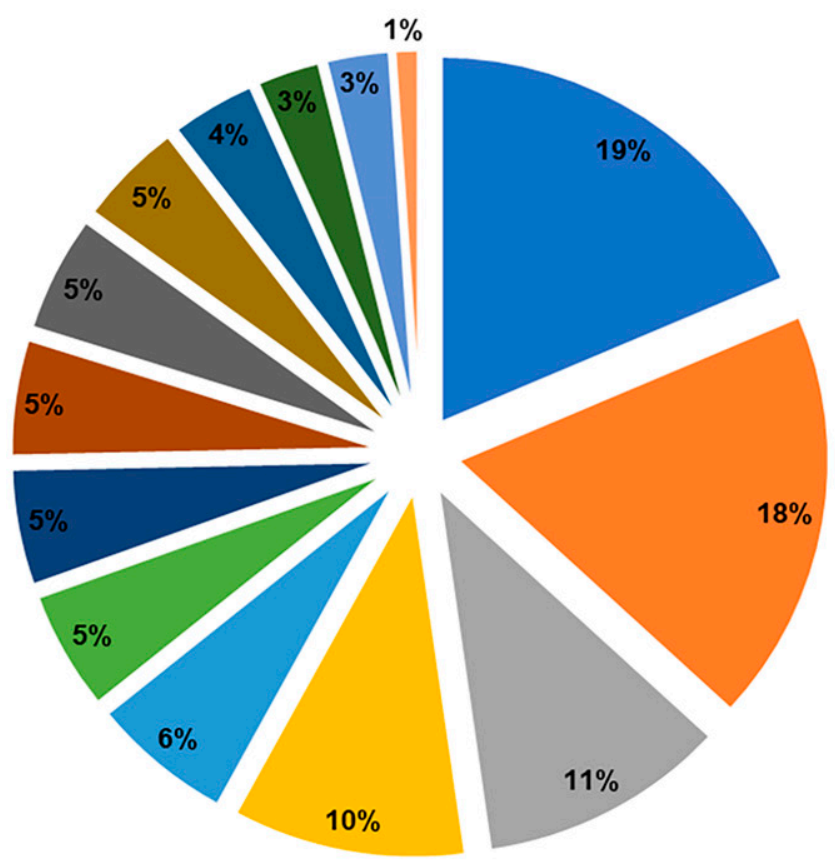

- Biosynthesis of amino acids

- Carbon metabolism

- Plant-pathogen interaction

- Oxidative phosphorylation

-2-Oxocarboxylic acid metabolism

• Pyruvate metabolism

- Arginine and proline metabolism

- Citrate cycle (TCA cycle)

- Carbon fixation in photosynthetic organisms

- Glycerolipid metabolism

- Pentose phosphate pathway

- Arginine biosynthesis

- Tropane, piperidine and pyridine alkaloid biosynthesis $=$ Glycosphingolipid biosynthesis

Fig. 2. Functional annotation of target genes of Brassica napus long noncoding RNAs A, Pathway enrichment analysis for the colocated targets and $\mathbf{B}$, pathway enrichment analysis for the coexpressed targets. 
that the majority of the coexpressed target genes belonged to primary metabolic pathways such as biosynthesis of amino acids (19\%) and carbon metabolism (18\%) (Fig. 2B). Some of the most significantly expressed lncRNAs in $\mathrm{R}$ and $\mathrm{S}$ plants and their predicted target genes are shown in Figure 3. A majority of the target genes regulated by these lncRNAs belonged to the plant defense-related pathways such as plant-pathogen interactions, phenylpropanoid biosynthetic pathways, plant hormone-signaling pathways, and primary metabolic pathways (Fig. 3). Genes involved in plantpathogen interactions include the calcium-dependent protein kinase, defensin, pathogen-related protein, and disease resistance protein (leucine-rich repeat $[L R R]$ receptor protein) genes (Fig. 3); and important genes from the phenylpropanoid biosynthetic pathway upregulated in $\mathrm{R}$ include caffeic acid 3-O-methyltransferase (CoMT), shikimate $O$ hydroxycinnamoyltransferase (HCT), and cinnamoyl alcohol dehydrogenase 7 (CADH7) (Fig. 3).

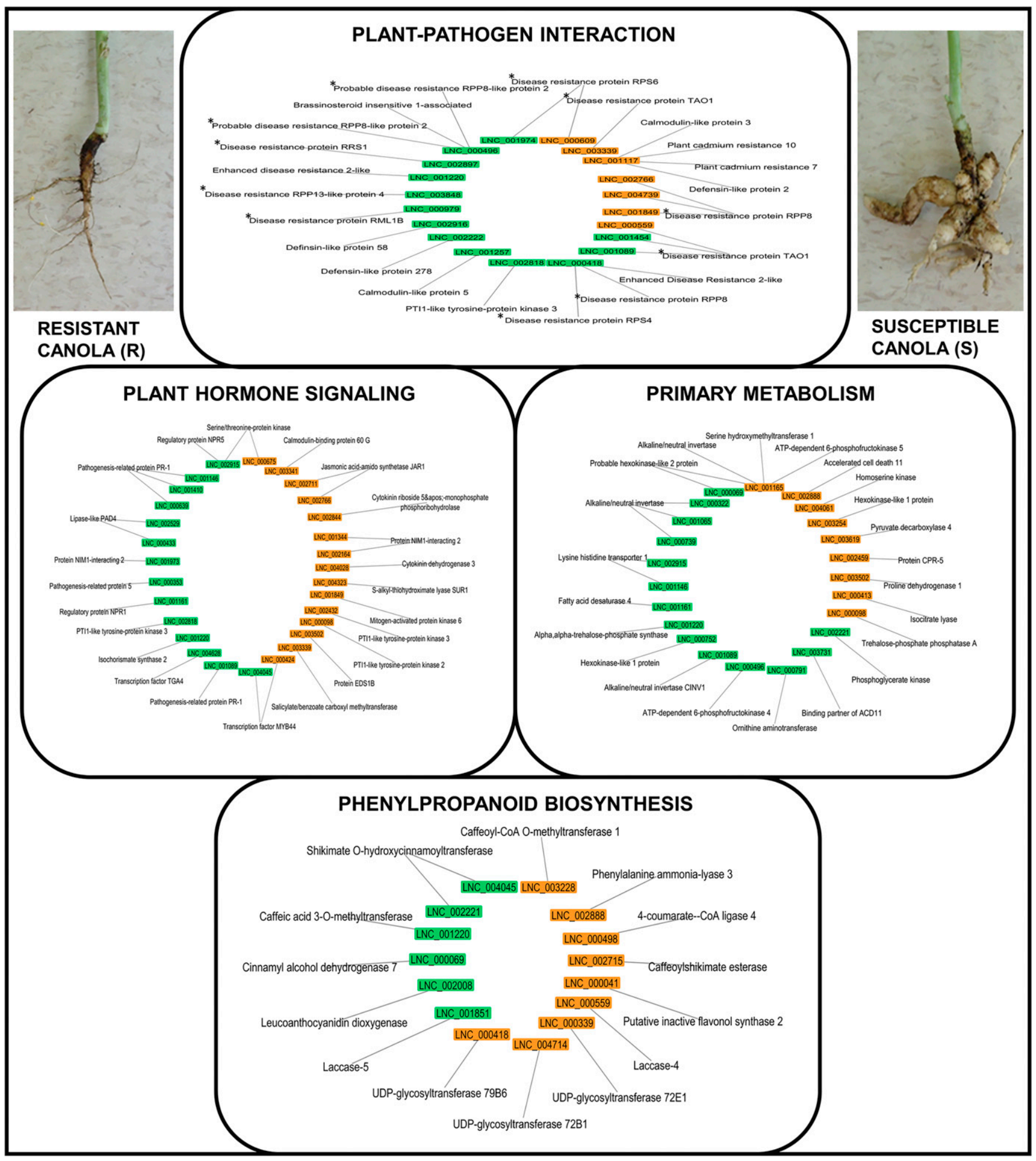

Fig. 3. Classification of long noncoding RNAs with the most significant differential expression and their predicted targets into the most represented functional groups. IncRNAs upregulated in R (green), IncRNAs upregulated in S (orange), and target genes with an asterisk are leucine-rich repeat protein genes. 


\section{DE genes of located on the A08 chromosome.}

Of the DE lncRNAs detected at 10 dpi, 24 lncRNAs were distributed on the chromosome A08 (Fig. 4); this chromosome has been reported to carry a genomic region conferring resistance to five $P$. brassicae pathotypes (Hasan and Rahman 2016). Among these, LNC_001065, LNC_001165, and LNC_001146 target the largest number of genes involved in plant defense (Fig. 4). While comparing their expression in the $\mathrm{R}$ and $\mathrm{S}$ plants, a greater number of the $24 \mathrm{DE}$ lncRNAs were found to be upregulated in the $\mathrm{S}$ plants as compared with the $\mathrm{R}$ plants. In addition, 8 of the 24 lncRNAs were detected only in the R plants; however, they showed a low and inconsistent expression among the three biological replicates (Fig. 5; Supplementary Table S4). Genes targeted by these 24 lncRNAs were found to be involved in primary metabolic pathways, plant-pathogen interactions, and secondary metabolite and phenylpropanoid biosynthetic pathways (Fig. 4).

All 24 DE lncRNAs, which were located on chromosome A08, were found to pair with the neighboring annotated target mRNAs (cis-regulation), and 21 of these were predicted to transregulate the target mRNAs. Among the 21 transregulated IncRNAs, LNC_001191, LNC_001126, LNC_001063, LNC_ 001117, LNC_001118, and LNC_001167 (Fig. 5) are known to be involved in the regulation of disease resistance protein genes distributed throughout the B. napus genome (Supplementary Table S5). Furthermore, LNC_001167 was predicted to be involved in the regulation of three different disease resistance genes encoding toll interleukin 1 receptor nucleotide-binding site LRR proteins, similar to those found in A. thaliana, including RPS4 (resistance to Pseudomonas syringae 4), RPP1 (recognition of Peronospora parasitica 1), and EDR2 (enhanced disease resistance 2). Expression of LNC_001063, LNC_001126, and LNC_001191 and could not be detected in the $\mathrm{R}$ plants whereas a 0.7 - to 2.7-fold increase in expression was observed for LNC_001117, LNC_001118, and LNC_ 001167 in the $\mathrm{S}$ plants.

When considering both lncRNAs and their targets located on chromosome A08, 15 of the abovementioned 24 lncRNAs were predicted to transregulate their target genes located on this chromosome (Fig. 5; Supplementary Table S6). No expression was observed for LNC_001063, LNC_001065, LNC_001089, LNC_001126, LNC_001146, LNC_001154, or LNC_001191 in the $\mathrm{S}$ plants, whereas a low expression was detected in the $\mathrm{R}$ plants (Supplementary Table S7). Based on KEGG pathway analysis, targets of these seven lncRNAs were found to be involved in the biosynthesis of secondary metabolites and in the primary metabolic pathways, including carbon and amino acid metabolism (Fig. 4). Among all of the lncRNAs located on chromosome A08, the greatest level of expression was observed for LNC_001165 in the S plants (Fig. 5; Supplementary Table S8). This lncRNA was predicted to transregulate 76 genes in the B. napus genome; however, 29 of these target genes are located on chromosome A08 and are known to be involved in carbon metabolism and biosynthesis of secondary metabolites.

\section{Quantitative real-time PCR validation for the expression of IncRNAs.}

To validate our findings from lncRNA-seq analysis and to further determine whether these genes are involved in defense responsive pathways, three lncRNAs from A08, three lncRNAs that are located on different chromosomes but have at least some of their target genes located on A08, and three lncRNAs were randomly selected for quantitative real-time (qRT)-PCR validation. While selecting the lncRNAs, only the lncRNAs which were predicted to have a maximum of four targets were used. Expression patterns of these nine lncRNAs were found to be similar in lncRNA-seq and qRT-PCR analysis (Fig. 6A); however, all of the target genes of an lncRNA did not follow the same pattern (Fig. 6B). For example, the three targets BnaA09g52970D, BnaC03g59610D, and BnaA08g06670D of LNC_003848 showed an expression profile similar to the results of lncRNA-seq; however, the fourth target (BnaA07g30470D) followed an opposite trend. It is probable that the target BnaA07g30470D is a false positive with $q$ value $>0.05$. A similar situation was also found in the case of the targets BnaC04g09450D, BnaC03g27800D, and BnaA07g23650D of LNC_001173. Interestingly, such anomalies were not observed in the cases where lncRNAs were predicted to have only one target, such as LNC_003279 and LNC_001192. Finally, the expression of two additional genes, BnaA10g17370D and BnaC09g40660D, showed excellent correlation between RNAseq (unpublished) and qRT-PCR results.

\section{B. napus IncRNAs as endogenous target mimics for microRNAs.}

By use of the RNA target analysis server psRNAtarget (Dai et al. 2018), with default parameters and maximum expectation of $3.5,15$ DE lncRNAs were identified which could possibly serve as target mimics of microRNAs (miRNAs0 (Supplementary Table S9). Among these, LNC_001191 and LNC_001163 were found to be located on chromosome A08 and as targets of the miRNAs bna-miRNA6028 and bnamiRNA824, respectively. Putative target AGL16 and the target mimic LNC_001163 of bna-miRNA824 are shown in Figure 7A. Among these two lncRNAs, LNC_001191 was expressed only in the R plants, and a greater level of expression was found for LNC_001163 (Fig. 5). qRT-PCR analysis also confirmed the upregulation of LNC_001163, bna-miRNA824, and its target AGL16 in the R plants (Fig. 7B). It was found that bnamiRNA6028 targets three lncRNAs (LNC_002844, LNC 001263, and LNC_001191); among these, the expression of LNC_002844 and LNC_001263 was downregulated in the R plants to a greater extent as compared with LNC_001191. From this data, six lncRNAs were predicted to target single miRNAs whereas four lncRNAs were predicted to target multiple miRNAs of the same family. The interaction analysis using Cytoscape v.3.7.0 (Fig. 8) depicts intricate interactions between the lncRNAs and miRNAs and mRNAs. Therefore, the abovementioned endogenous target mimics (eTMs) identified in response to Plasmodiophora brassicae infection were predicted to conserve the mRNA targets by disrupting the miRNAmRNA interactions.

\section{IncRNAs as potential miRNA precursors.}

IncRNAs can also function as precursors for miRNA and siRNA, playing important roles in modulating gene expression and, thus, implicating their roles in diverse biological functions in plants. In this study, 8 of the $530 \mathrm{DE}$ lncRNAs were identified to be possible precursors of miRNA (Supplementary Table S10). For instance, LNC_001710 seems to harbor $100 \%$ of the stem-loop sequence of miRNA-160a; and LNC_000265 and LNC_004360 were predicted to be the precursors for miRNA169n and miRNA166a, respectively (Fig. 9), and were expressed at higher levels in the $\mathrm{R}$ plants. Similarly, miRNA166a was also predicted to arise from LNC_00563; however, expression of this IncRNA was not significantly different between the $\mathrm{R}$ and $\mathrm{S}$ plants. The miRNA166 is a family of six miRNAs in B. napus, of which miRNA166a (LNC_004360) and miRNA166e (LNC_001636) were represented in this study.

\section{Natural antisense transcripts.}

In total, 59 natural antisense transcripts (NATs) was identified to be regulating their corresponding sense transcripts in response to infection by $P$. brassicae at 10 dpi (Supplementary 


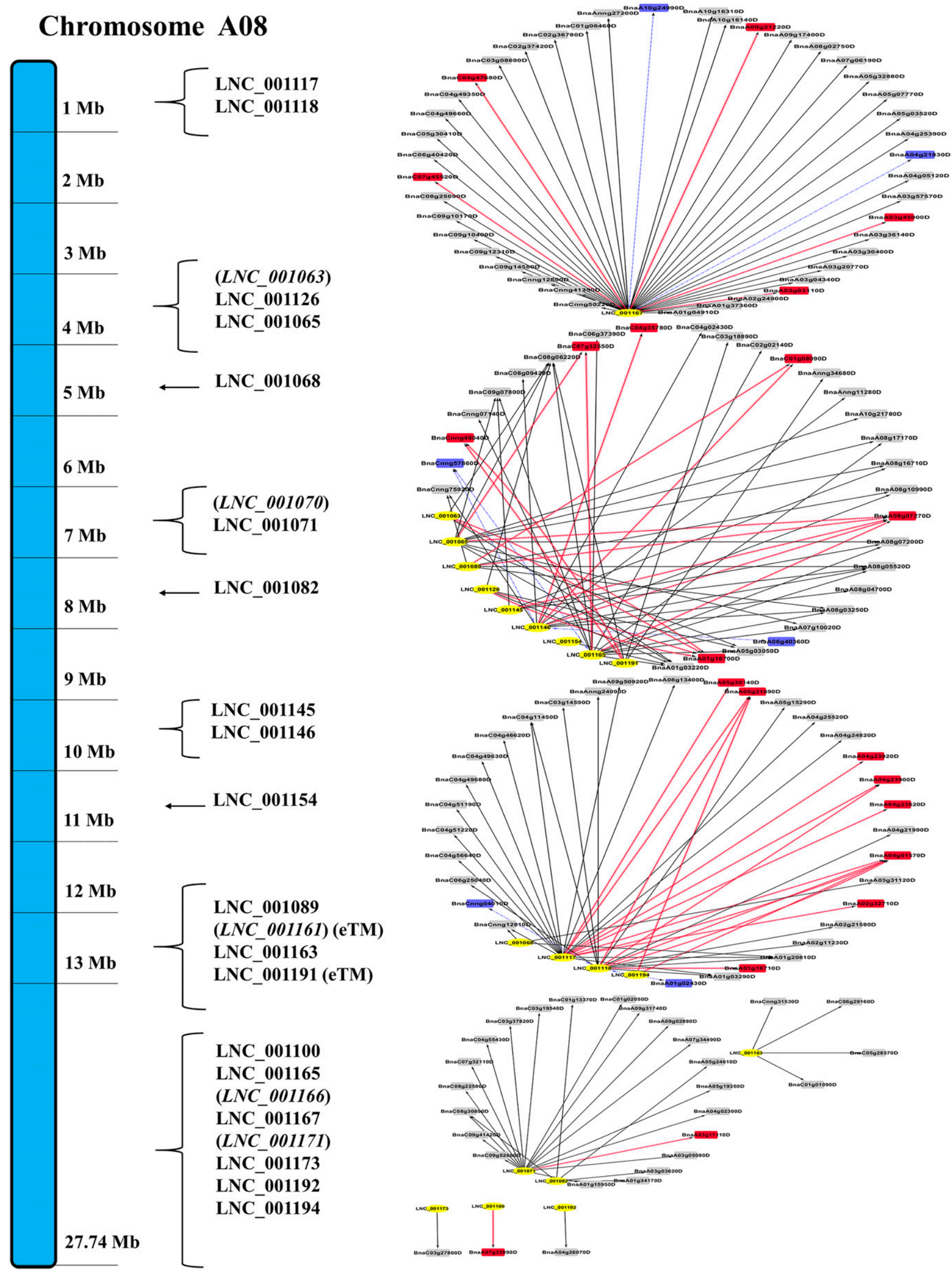

Fig. 4. Long noncoding RNAs (lncRNAs) on chromosome A08 and their interaction with target genes in Brassica napus genome involved in plant defense. lncRNAs not regulating any target genes involved in plant defense are shown in italics and parentheses. Yellow ovals $=$ lncRNAs on chromosome A08, red squares $=$ target genes involved in plant-pathogen interaction, blue squares $=$ target genes involved in plant hormone signaling, gray squares $=$ target genes involved in primary and secondary metabolism, and eTM = endogenous target mimic. 
Table S11). All of these NATs were validated in silico by determining the thermodynamics of RNA-RNA interaction (Hofacker 2009; Lorenz et al. 2011). Based on differential expression of the lncRNAs, 38 NATs were identified. After filtering the 38 NATs based on expression of their sense transcripts in the RNA-seq data and potential pathogen defensive functions of the genes, 18 NATs were selected, of which 4 were validated using qRT-PCR (Figure. 10). The optimal secondary structure formation happens when thermodynamic free energy is minimum; such minimum free-energy structures of these four lncRNA-mRNA dimerized pairs, predicted using RNAcofold (Lorenz et al. 2011), are shown in Figure 10.

\section{DISCUSSION}

CR disease is known to cause significant yield loss in Brassica spp., including vegetables and oilseed crops. Significant achievements have been made in combating this disease through the development of $\mathrm{R}$ cultivars (Piao et al. 2004; Rahman et al. 2014); however, the molecular mechanisms involved in the host plant resistance have not been well understood. Results from our study indicate the possible involvement of lncRNAs in mediating resistance in B. napus canola lines carrying resistance introgressed from rutabaga. We did not find evidence of infection in the roots at 6 dpi in both $\mathrm{R}$ and $\mathrm{S}$ plants; however, infection was observed in both $\mathrm{R}$ and $\mathrm{S}$ plant roots at 10 dpi. In contrast, Dobson et al. (1983) observed root hair infection in Chinese cabbage at 1 dpi. However, Asano et al. (2000) detected initial infection in turnip at 4 dpi with rapid increase up to $6 \mathrm{dpi}$; similar results have also been reported by Hwang et al. (2012) in canola. Thus, it is evident that the stage of root hair infection may be dependent on the type of plant material and the method of inoculation which, in the present study, was injection

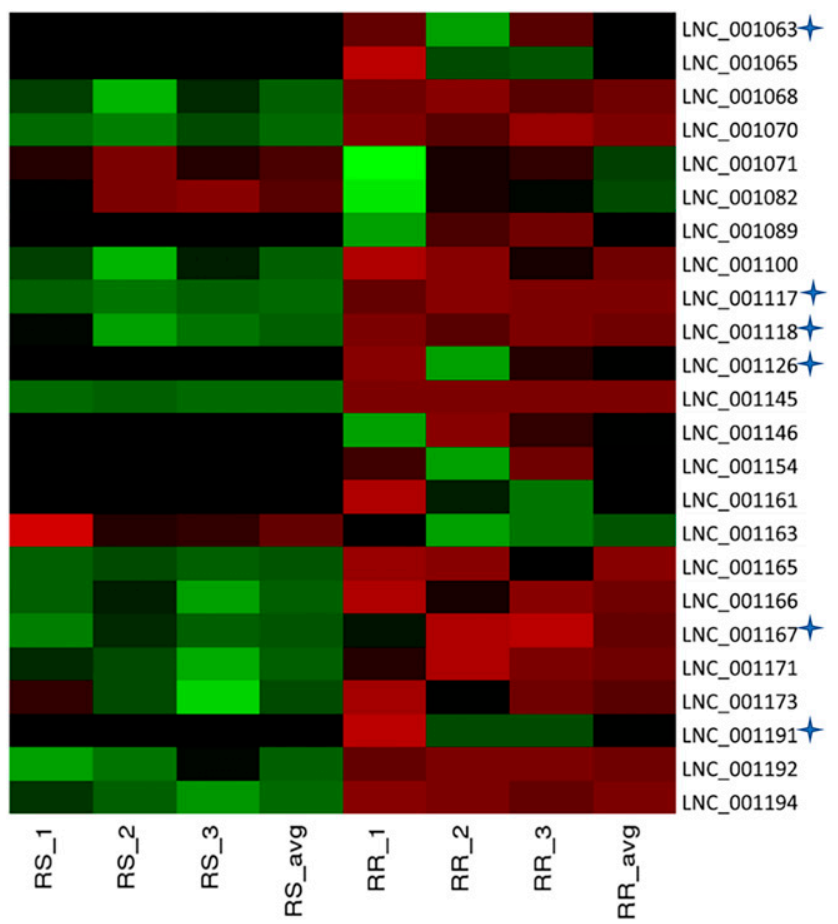

Fig. 5. Differentially expressed long noncoding RNAs (lncRNAs) from chromosome A08 of Brassica napus. Expression values are scaled from +2 (green) to -2 (red); black indicates no expression. S_1, S_2, and S_3 are the biological replicates of clubroot (CR)-susceptible $\bar{B}$. napus and R_1, R_2, and $\mathrm{R} \_3$ are the biological replicates of CR-resistant B. napus. IncRNAs with asterisks are known to target genes encoding disease resistance proteins. of spore suspension into the soil (direct inoculation). A noticeable reduction in the formation of primary plasmodia was found in the $\mathrm{R}$ plants, with no infection in the newly emerging roots at $14 \mathrm{dpi}$, whereas gall formation had already begun in the $\mathrm{S}$ plants at this stage. Detection of infection at an early stage in the $\mathrm{R}$ plants could be related to the plant development; an influence of the developmental stage of the host plant on the pathogen's ability to cause infection has been suggested by Fei et al. (2016). Thus, our study suggests that, despite microscopically visible infection that occurs in the $\mathrm{R}$ plants at their early growth stage, the resistance genes restrict further development of the disease.

Most of the 530 DE lncRNAs detected in this study were from the intergenic regions (lincRNAs); these lncRNAs showed considerably greater expression as compared with the expression of the antisense lncRNAs (NATs), which is consistent with the results from other plant species (Li et al. 2014; Wang et al. 2014; J. Zhang et al. 2018). The lincRNAs regulate plant responses to CR by remodeling epigenetic and chromatin architecture, stabilization and localization of proteins and RNA molecules by tethering to them and transcription regulation involving enhancer-associated activity, and cis- or transmediated gene activation (Kopp and Mendell 2018; Kornienko et al. 2013; Nejat and Mantri 2018; Zaynab et al. 2018). To understand the function of DE lincRNAs under CR stress, we analyzed the protein coding genes coexpressed with these lincRNAs. This putative functional prediction relied on the development of a coexpression network. The coexpression analysis indicated that $425 \operatorname{lncRNAs}$ were predicted to have transregulated targets whereas all DE 530 lncRNAs were predicted to have cis-regulated targets. KEGG pathway analysis revealed that a majority of transregulated target genes belong to carbon (18\%) and amino acid biosynthesis pathways (19\%); however, most of the cis-regulated target genes belong to the phenylpropanoid biosynthetic pathway (15\%) and are involved in plant-pathogen interactions (15\%). This suggests that the energy required to fuel the defense in $\mathrm{R}$ plants is probably provided by the carbon metabolism. Also, it is likely that biosynthesis of specific amino acids which are upregulated or reduced are involved in the plant defense (Rojas et al. 2014). However, more comprehensive studies are required to verify this hypothesis.

IncRNAs, like NATs, are also known to mediate the regulation of the corresponding sense transcripts in a concordant or discordant manner at a transcriptional and posttranscriptional level (Rai et al. in press; Wight and Werner 2013). In B. napus, 111 NATs were detected in response to $S$. sclerotiorum infection at 24 and $48 \mathrm{~h}$ postinoculation (hpi) (Joshi et al. 2016). In contrast, we observed very limited expression of the NATs in response to $P$. brassicae infection, which is possibly due to the fact that this type of lncRNA can be expressed in a spatial and temporal manner (Wang et al. 2014). Some of the NATs such as COOLAIR (Ietswaart et al. 2017), cis NAT $T_{P H O 1 ; 2}$ (Jabnoune et al. 2013), SHO lnc-NAT (Zubko and Meyer 2007), and $T L$ IncRNA (Liu et al. 2018) are involved in the regulation of plant morphology and biotic or abiotic responses. In this study, we found that LNC_000418, LNC_000789, LNC_000674, and LNC_000562 are antisense to the protein-coding genes Raclike GTP-binding protein, defensin-like protein 2, flavone 3dioxygenase 3-like protein, and WRKY transcription factor 12, respectively; all four genes are directly or indirectly involved in plant secondary metabolism. Thus, considering the function of the predicted target genes of IncRNA, we suggest that primary and secondary metabolic pathways are also regulated by NATs and may play a role in the resistance to $P$. brassicae.

Most of the target genes identified in this study belong to the primary and secondary metabolic pathways, and include the genes involved in amino acid biosynthesis, carbon metabolism, 
phenylpropanoid biosynthesis, and biosynthesis of secondary metabolites, indicating their probable association with the CR disease (Fig. 3). The importance of phenylpropanoid biosynthesis has already been demonstrated in response to biotic stress because it is known to generate an array of secondary metabolites involved in host-pathogen interactions (Caretto et al. 2015; Vogt 2010). Genes encoding proteins such as phenylalanine ammonia lyase (Vogt 2010), laccase 4, laccase 7 (Hu et al. 2018), and UDP-glycosyltransferases (Le Roy et al. 2016) have been implicated to play a significant role in phenylpropanoid biosynthetic pathways, and they have also been identified as targets of IncRNAs in our study. It is known that caffeoyl-CoA O-methyltransferase 1, CoMT, HCT, and CADH7 play critical roles in the biosynthetic pathway for lignification (Harakava 2005); among these genes, CoMT, HCT, and CADH7 were found to be upregulated in the R plants at 10 dpi (Fig. 3). This suggests that the upregulation of this pathway prevents the spread of the $P$. brassicae in the roots of R plants and, thus, contributes to the decline in disease progression. Rojas et al. (2014) suggested that induction of primary metabolic pathways may be equally important because they can modulate signal transduction cascades influencing the plant defenses. Several

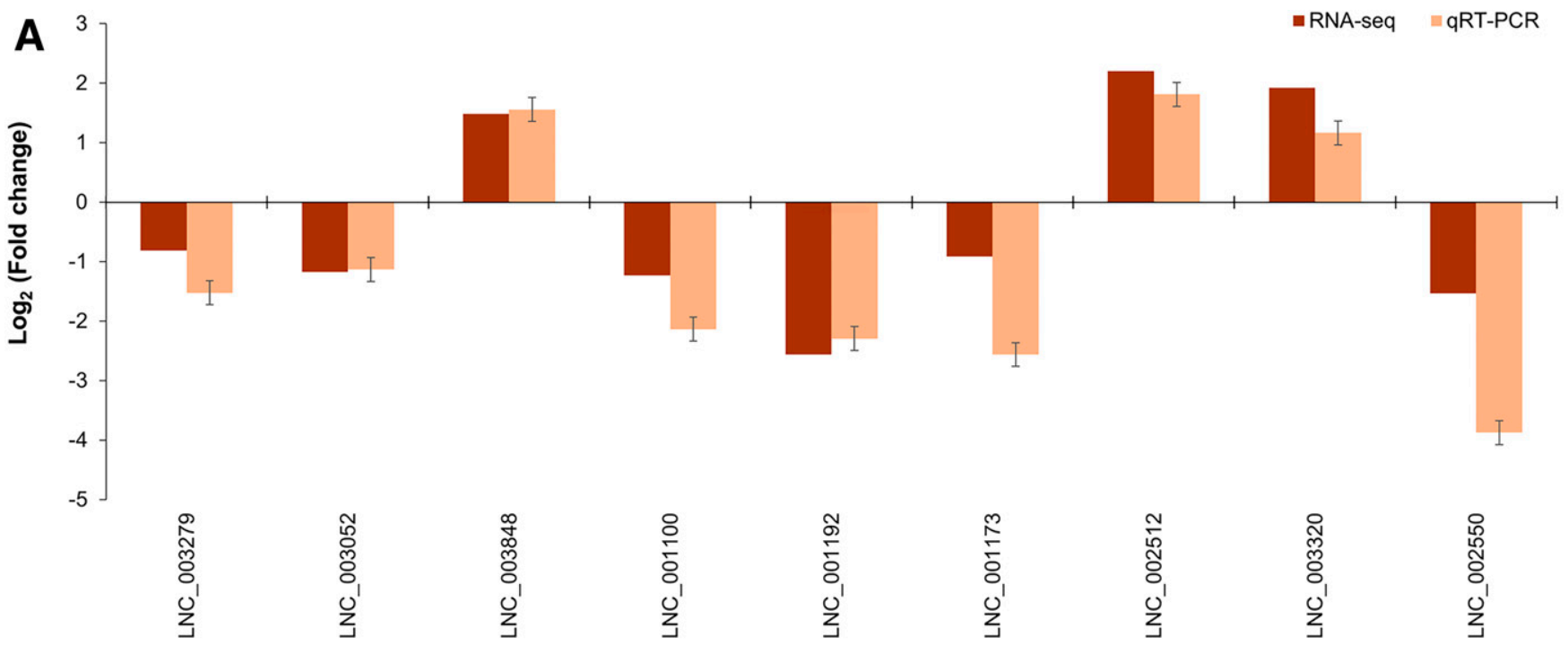

LncRNAs

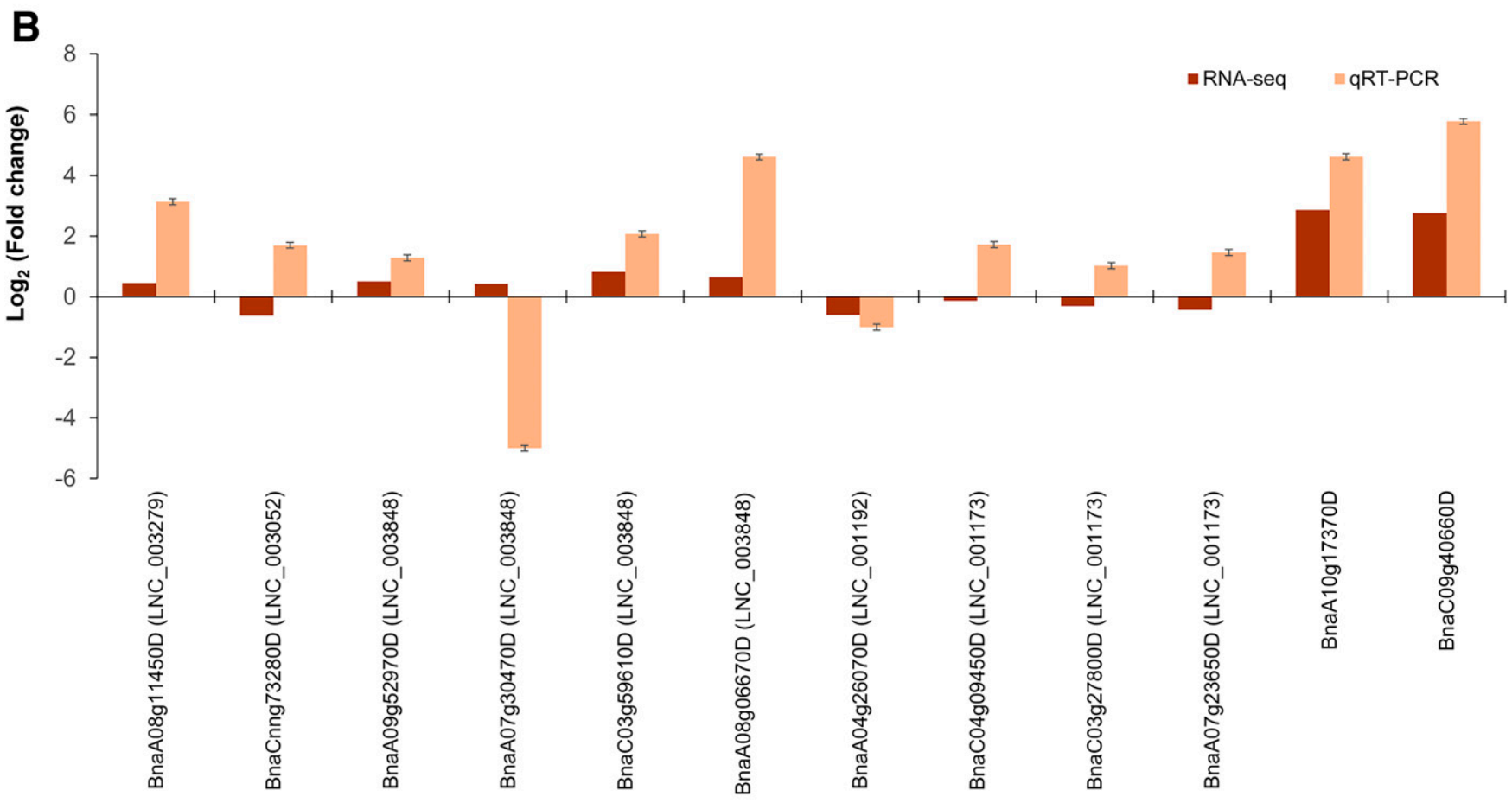

\section{Predicted targets of IncRNAs}

Fig. 6. A, Quantitative real-time (qRT)-PCR validation of pathogen-induced nine long noncoding RNAs (lncRNAs) detected by lncRNA-seq. B, qRT-PCR validation of lncRNAseq data for 10 targets predicted by correlation of expression with lncRNAs and two randomly selected genes. The expression of lncRNAs and target genes was normalized to UBC9 (endogenous control) and lncRNAs regulating their respective targets are indicated within parentheses. 
researchers have suggested that carbon skeletons synthesized during photosynthesis are used for growth (primary metabolism) or defense responses (secondary metabolism) (Caretto et al. 2015). Tayeh et al. (2014) reported that external application of the disaccharide sugar trehalose (consisting of two molecules of glucose; it is also known as mycose or tremalose) increases the expression of $P R$ protein genes in Arabidopsis, and external application of glucose upregulates $P R 1$ and $P R 5$ (Xiao et al. 2000). Trehalose is considered an inducer of plant defense to biotic and abiotic stresses (Tayeh et al. 2014). In this study, we

\section{A}

\section{Bna-miR824}

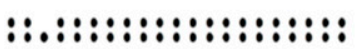

AGL16 5'750 UCUCUUCUCACAAAUGGUCUA 770 3'

\section{Bna-miR824 \\ 3' 21 AggGaAGAGUGUUUACCAGAU 1

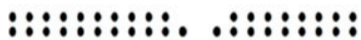 \\ LNC_001163}

B

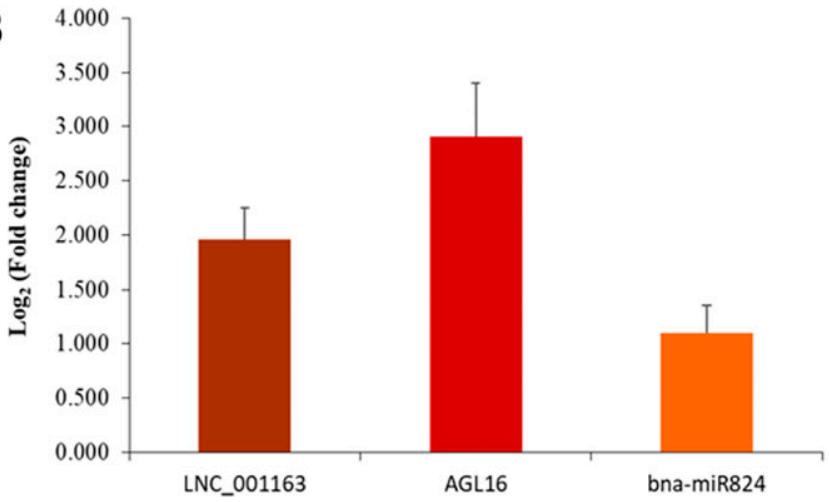

Fig. 7. Putative endogenous target mimic (eTM) LNC_001163 of AGL16 inhibiting bna-miR824 in response to clubroot infection in Brassica napus. A, Predicted base-pairing interactions between bna-miR824 and AGL16 and eTM long noncoding RNA LNC_001163 and bna-miR824. B, Quantitative real-time PCR results for LNC_001163, AGL16, and bna-miR824.
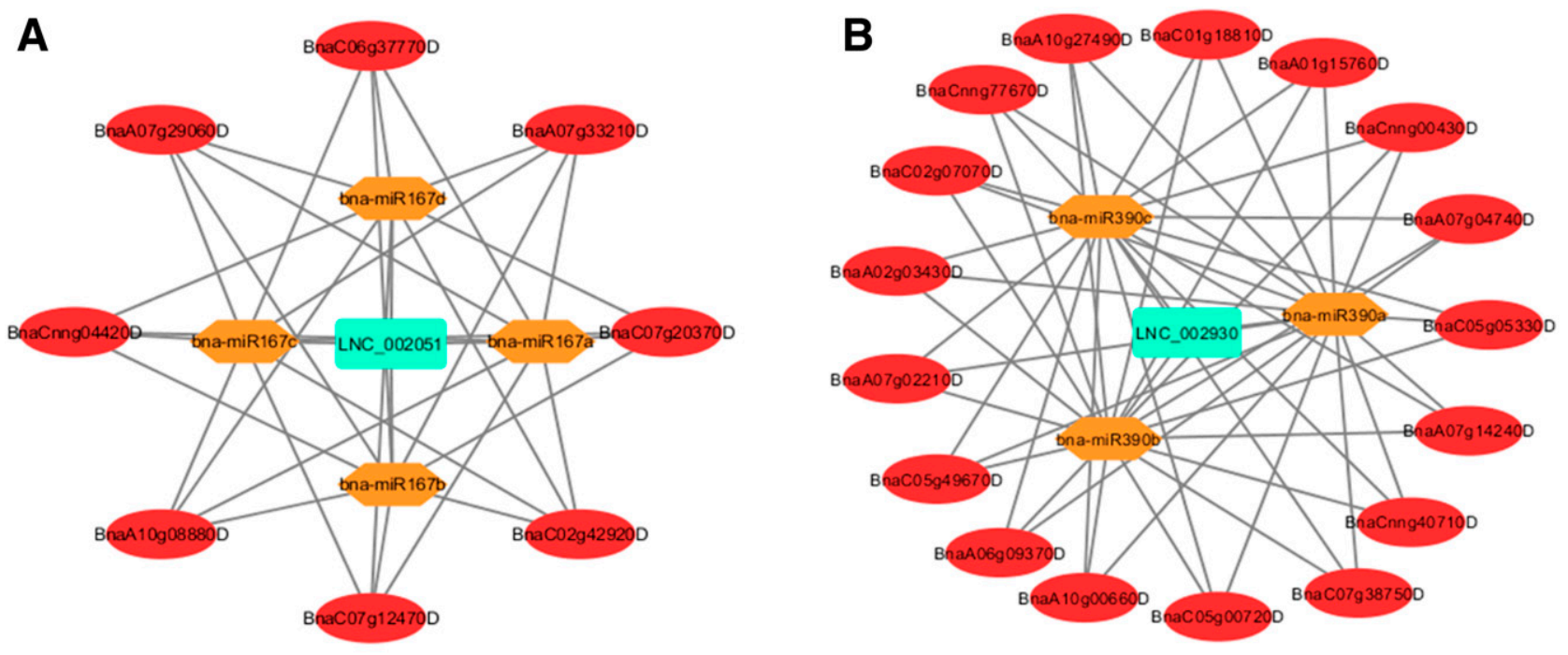

C
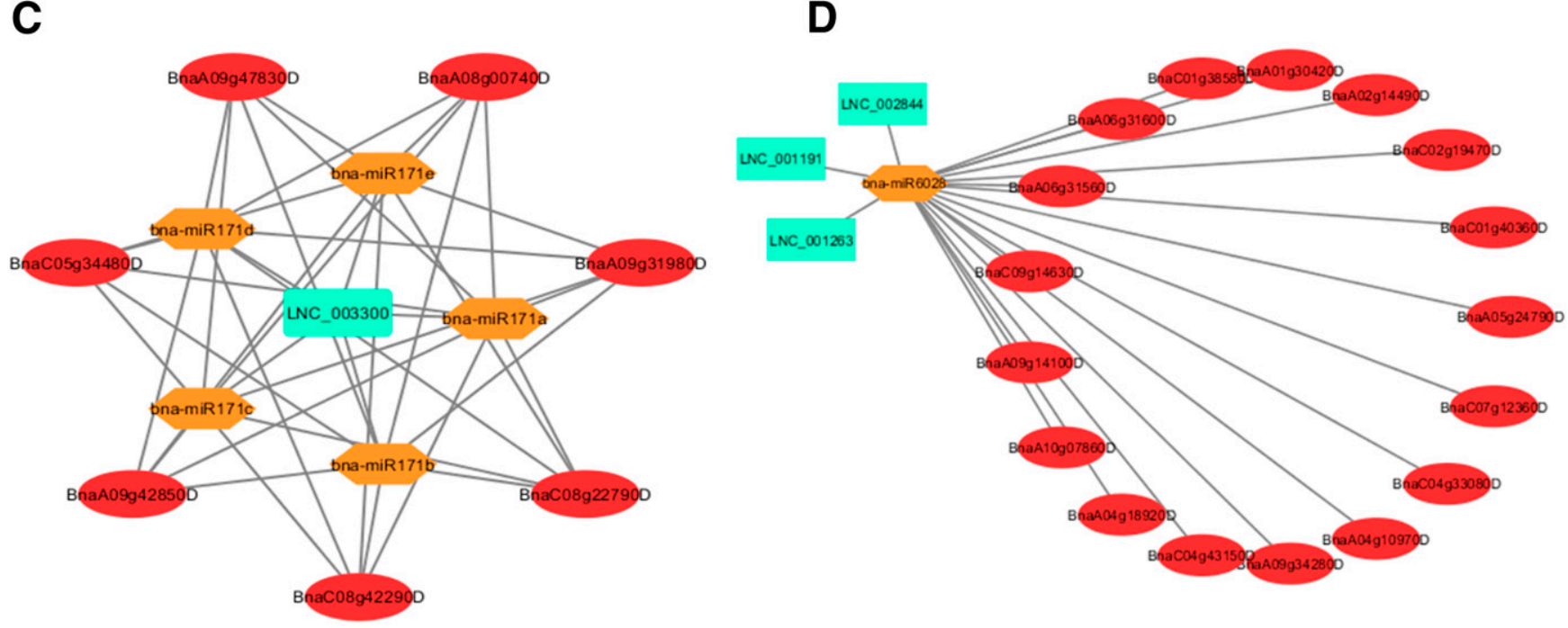

Fig. 8. A, B, and C, Interaction networks of a single long noncoding RNAs (IncRNAs), microRNA (miRNA) family, and the mRNAs; and D, interaction network of multiple lncRNAs with miRNA and mRNAs. 
found an increased expression of LNC_002915, which is predicted to regulate an $\alpha, \alpha$-trehalose phosphate synthase 10 (TPS10), and a considerable decrease in the expression of LNC_00675 and its targets regulating the synthesis of trehalose phosphate phosphatase A. In addition, alkaline or neutral invertase and other similar proteins are involved in carbon metabolism and are known to cleave sucrose into glucose and fructose for energy production (Ehness et al. 1997) and we observed upregulation of these and similar genes in our study (Fig. 2). Functional research with these lncRNAs can provide further insight into the regulatory mechanism involved in primary metabolism and, eventually, in pathogen defense.

Hasan and Rahman (2016) mapped the CR resistance of rutabaga, which was introgressed into oilseed $B$. napus, on chromosome A08. By use of this resistance, we identified eight lncRNAs showing expression only in the R plants; however, expression these lncRNAs was low and not very consistent. Kopp and Mendell (2018) suggested that many lncRNAs can exhibit low expression in plants but still play an important role. Taking this into account, it can be assumed that these lncRNAs might play an important role in $\mathrm{CR}$ resistance through regulating protein coding genes or proteins by tethering to them (Fig. 4). Coexpression analysis of LNC_001063, LNC_001191, and LNC_001126 had predicted 8, 46, and 47 target genes, respectively, in the $B$. napus genome, and each lncRNA was found to regulate a different resistance $(R)$ gene as well as genes directly or indirectly modulating primary metabolism (Fig. 4). Another lncRNA (LNC_001167) was also predicted to regulate three $R$ genes and 35 other genes modulating primary metabolism and phenylpropanoid biosynthesis (Fig. 4).

We further validated the relative expression of the selected lncRNAs and their target genes based on their direct or indirect association with chromosome A08. For instance, relative expression exhibits a positive correlation and LNC_003848 was upregulated in the $\mathrm{R}$ plants and exhibits a positive correlation with the expression of the target gene Transparent Testa 12 (BnaA08g06670D) located on chromosome A08. Genes transparent testa 12 as well as gibberellin 2- $\beta$-dioxygenase 2 (BnaC03g59610D) are known to be involved in flavonoid and plant hormone biosynthesis and were found to be transregulated by LNC_003848. Flavonoids are known to play important roles such as protecting the plants from UV radiation, pathogen attack, and photo-oxidative stress (Falcone Ferreyra et al. 2012; Treutter 2006). The specific roles of these lncRNAs, their target genes, as well as the regulatory mechanism remain to be validated.

IncRNAs have also been hypothesized to form functional networks with miRNAs and mRNAs by functioning as either target mimics of miRNAs or precursors to miRNAs (Meng et al. 2012; Wu et al. 2013). Results from our study indicate that LNC_001163 and LNC_001191, located on chromosome A08, could serve as an eTM of bna-miR824 and bna-miR6028, respectively. Kutter et al. (2007) reported that bna-miR824
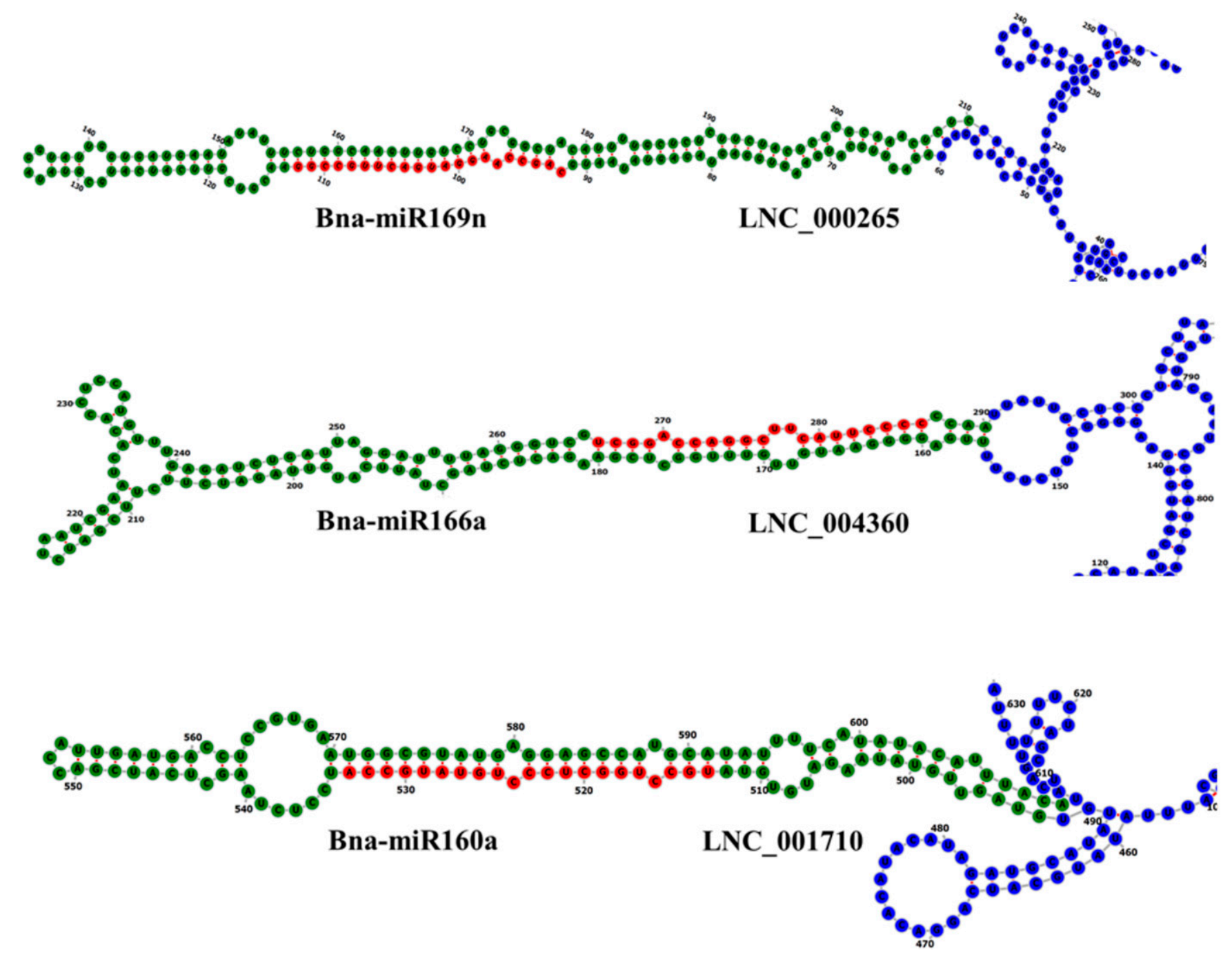

Fig. 9. Representative predicted long noncoding RNA functioning as microRNA (miRNA) precursor sequences. 
targets the MIKC-type MADS box protein agamous-like 16 (AGL16), and our results indicated that the expression of AGL16 and the predicted target mimic LNC_001163 of bnamiR824 were upregulated and positively related to each other in response to $\mathrm{CR}$ infection (Fig. 7B). Interaction analysis among the mRNAs, miRNAs, and lncRNAs suggested multiple and complex sets of interactions, including instances where a single lncRNA interacts with an miRNA family (Fig. 8A, B, and $\mathrm{C}$ ) and a single miRNA interacts with multiple lncRNAs and mRNAs (Fig. 8D). Shumayla et al. (2017) also reported interaction of 1,047 lncRNAs with 222 miRNAs, which further interacted with 209 mRNA transcripts with various roles in

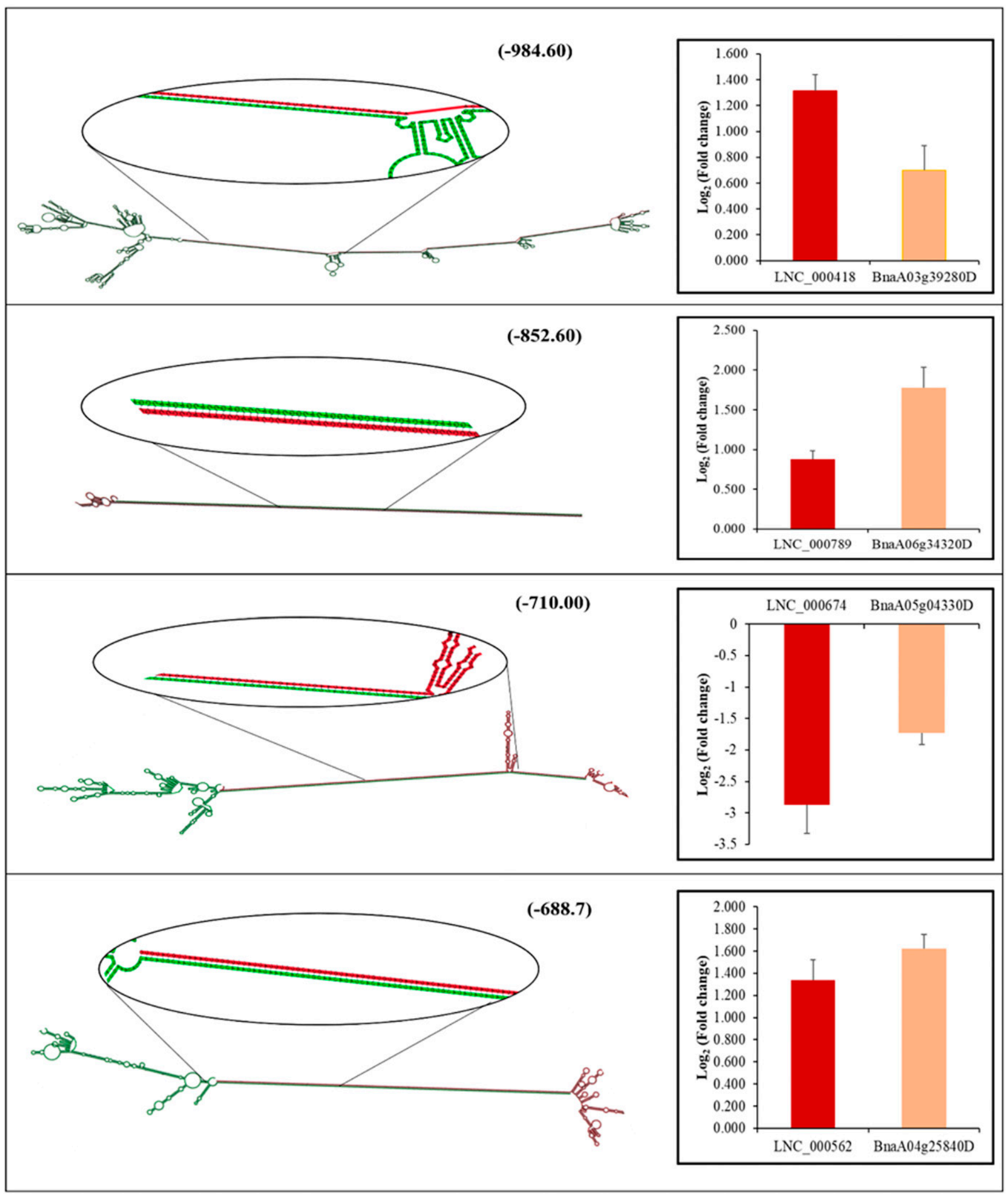

Fig. 10. Minimum free energy (MFE) structures for representative long noncoding RNA-mRNA pairs. MFE for each secondary structure is given in the bracket. Quantitative real-time PCR results for each pair are shown on the right side. 
Triticum aestivum. We also detected eight lncRNAs in the present study to be the precursors of five miRNAs. For instance, LNC_000265 was observed to be upregulated in the R plants, and this seems to be the precursor for bna-miR169 in B. napus (bna-miR69n) (Fig. 9). bna-miR169 has been reported to be upregulated in response to drought in tomato (Zhang et al. 2011) and salt and drought stress in maize (Luan et al. 2014). Additionally, LNC_004360, a putative precursor of miRNA166a, was significantly expressed in the R plants (Fig. 9). The expression of miRNA166 has also been associated in response to Botryosphaeria dothidea in Populus trichocarpa (Zhao et al. 2012). Recently, J. Zhang et al. (2018) reported seven lncRNAs from Brassica oleracea and 15 lncRNAs from $B$. rapa, which are precursors of nine and 19 miRNAs belonging to eight and 15 miRNA families, respectively. Xin et al. (2011) and Joshi et al. (2016) demonstrated that lncRNAs can serve as precursors of miRNAs in plants and are involved in the regulation of plant responses to biotic stress. Several researchers such as Chauhan et al. (2017), Koroban et al. (2016), and Kumar (2014) also have summarized the functions of miRNAs in biotic and abiotic stresses, and results from our interaction analyses also lend credence to additional layers of gene regulation which may result from interactions of lncRNAs-miRNA and lncRNAstarget miRNAs.

\section{Conclusion.}

Using lncRNAseq analysis, we have, for the first time, identified 530 DE lncRNAs in B. napus in response to Plasmodiophora brassicae. By comparing the response of the lncRNAs in the R and S plants, we have suggested a potentially important role in mediating resistance to $P$. brassicae. qRTPCR analyses confirmed the expression trend of the lincRNAs and NATs with that of lncRNA-seq. Of the 530 lncRNAs, 16 DE lncRNAs were predicted to function as eTMs of miRNAs and eight DE lncRNAs as the precursor of miRNAs. Of the 24 DE lncRNAs from chromosome A08, eight showed low expression only in the R plants. Pathway analyses showed that the majority of the target genes regulated by these lncRNAs belong to the pathways involved in plant-pathogen interaction, plant hormone signaling, and primary-secondary metabolism. In this regard, the IncRNAs and their targets from A08 provide valuable information because this chromosome is associated with resistance to multiple pathotypes. Moreover, the knowledge of the $P$. brassicae-responsive lncRNAs gained from this research can be used for knock-down or overexpression studies to understand their molecular mechanisms and regulatory functions and may be useful in the development of markers in a breeding program for $\mathrm{CR}$ resistance.

\section{MATERIALS AND METHODS}

\section{Plant material.}

A doubled-haploid $(\mathrm{DH})$ population derived from $\mathrm{F}_{1}$ generation of Rutabaga-BF $\times$ UA AlfaGold was used in this study. The parent Rutabaga-BF is a CR-R inbred line derived from the rutabaga ( $B$. napus var. napobrassica) cultivar Brookfield through self pollination of single plants (Hasan and Rahman 2016), while UA AlfaGold is an open-pollinated CR-S springtype B. napus canola cultivar (Rahman 2016). The details of the development of the $\mathrm{DH}$ lines and phenotyping for resistance to $\mathrm{CR}$ disease have been described previously (Hasan and Rahman 2016).

\section{Microscopy analysis for primary infection by $P$. brassiceae.}

To determine the time of primary infection by $P$. brassicae and the development of disease symptoms, 12 CR-R and 12
$\mathrm{CR}-\mathrm{S} \mathrm{DH}$ lines were grown in a greenhouse at 22 and $15^{\circ} \mathrm{C}$ (day and night, respectively) with a 16 -h photoperiod. In total, eight plants of these lines were grown in 72-cell trays ( 52 by 26 by $5 \mathrm{~cm}$, length by width by depth, respectively) filled with Sunshine Professional Growing Mix (Sun Gro Horticulture Canada Ltd.). Ten-day-old seedlings were inoculated with $P$. brassicae single-spore isolate SACAN-ss1 of pathotype 3 (Williams 1966) (obtained from Dr. Stephen Strelkov, University of Alberta) following a direct inoculation method. The details of inoculum preparation and inoculation procedure have been described in a previous study (Hasan and Rahman 2016). Briefly, the inoculum was prepared by crushing the galls using sterile water and the homogenized spore suspension was filtered through multilayered cheesecloth. The spore concentration was adjusted to $1 \times 10^{7}$ spores $/ \mathrm{ml}$ and inoculation was done by injecting $1 \mathrm{ml}$ of the spore suspension into the soil near the root of the seedlings (Voorrips and Visser 1993). Root samples of the inoculated seedlings were collected at 0 hpi and 6, 10, 14, 18, and 22 dpi. Uninoculated controls were also included in the study.

The roots were gently washed with water, cut into 10 - to 15-mm segments, and fixed in a solution of formalin, acetic acid, and alcohol comprising $5 \mathrm{ml}$ of commercial formalin (40\% formaldehyde solution), $5 \mathrm{ml}$ of glacial acetic acid, and $90 \mathrm{ml}$ of $50 \%$ ethyl alcohol (Yeung and Saxena 2005) at room temperature for $24 \mathrm{~h}$ (Verma et al. 2014). Following this, samples were dehydrated in a series of ethanol dilutions, gradually followed by the treatment with the toluene (clearing agent) using Leica TP1020 tissue processor (Leica Biosystems). The tissues were then infiltrated with ParaplastH (Leica Biosystems) and cast into rectangular blocks. Longitudinal sections of roots, $10 \mathrm{~mm}$ thick, were prepared using A0 Rotary microtome (Leica Biosystems) and affixed to glass slides. The samples on the glass slides were deparaffinated with toluene, rehydrated using a graded series of ethanol, stained with Harris Hematoxylin, and counterstained with Eosin Y (Leica Biosystems). After dehydrating again with $100 \%$ ethanol and toluene, the slides were mounted with DPX mounting media (Sigma-Aldrich). The sections were viewed with a Zeiss Axioscope, photographed with Zeiss Axiocam, and analyzed using AxioVision software (Verma et al. 2014).

\section{Plant materials for IncRNA-seq analysis.}

In all, $12 \mathrm{R}$ and $12 \mathrm{~S}$ DH lines were used for this study. In total, 192 seedlings of the $24 \mathrm{DH}$ (12 each of $\mathrm{R}$ and $\mathrm{S}$ ) lines $(24 \times 8=192)$ were grown in the abovementioned 72 -cell trays filled with Sunshine Professional Growing Mix. Seedlings were inoculated with $P$. brassicae single-spore isolate SACAN-ss1 pathotype 3, as described above. The inoculated plants were maintained in a greenhouse at 22 and $15^{\circ} \mathrm{C}$ (day and night, respectively) with a 16-h photoperiod, and the soil was kept saturated for successful infection and disease development. The experiment was repeated three times as biological replicates.

\section{RNA isolation, library preparation, and sequencing.}

For RNA isolation, roots of three seedlings from each of the $12 \mathrm{R}$ and $12 \mathrm{~S} \mathrm{DH}$ lines were harvested at $10 \mathrm{dpi}$ as bulk of $\mathrm{R}$ and $\mathrm{S}$ samples, respectively; frozen in liquid nitrogen; and stored at $-80^{\circ} \mathrm{C}$ until further use. The remaining plants of the $\mathrm{R}$ and S DH lines were grown to maturity and phenotyped for resistance in order to confirm the resistance or susceptibility of the DH lines.

Total RNA from the $\mathrm{R}$ and $\mathrm{S}$ bulks was extracted using TRIzol reagent (Invitrogen) and the Qiagen RNeasy Plant Mini Kit (Qiagen) as per the manufacturer's instructions. Root 
samples were homogenized into a fine powder and $100 \mathrm{mg}$ of this tissue powder was treated with $1 \mathrm{ml}$ of TRIzol in an Eppendorf tube. The mixture was centrifuged at 9,425 relative centrifugal force (RCF) for $15 \mathrm{~min}$ at $4^{\circ} \mathrm{C}$ and the supernatant was collected in a new tube. To achieve phase separation, $200 \mu \mathrm{l}$ of chloroform was added to the supernatant, mixed vigorously, incubated at room temperature for $5 \mathrm{~min}$, and centrifuged at 9,425 RCF. The aqueous layer was collected in a new tube without disturbing the interface layer containing DNA and $0.5 \mathrm{ml}$ of chilled $95 \%$ ethanol was added and mixed. The sample was then transferred to an RNeasy minispin column (pink) of the Qiagen RNeasy Plant Mini Kit and the next steps were carried out as per the manufacturer's instructions. The quality and quantity of total RNA was determined using a NanoDrop ND-2000 spectrophotometer (Thermo Fisher Scientific). The integrity of the total RNA was determined on an Agilent Tapestation using RNA Screen Tape assay following the manufacturer's instructions (Agilent Technologies). The RNA samples with RNA integrity number greater than nine were used for further analysis.

In total, six libraries - three R (each sample with bulks of 12 plants) and three $S$ (each sample with bulks of 12 plants) - were prepared and $4 \mu \mathrm{g}$ of RNA of each sample was used for library preparation. Ribosomal RNA (rRNA) was removed from the samples using an Epicentre RIbo-zero rRNA Removal Kit (Epicentre) and strand-specific libraries were prepared using a NEB Next Ultra Directional RNA Library Prep Kit (NEB) following the manufacturer's instructions. RNA library sequencing was carried out using the Illumina Hiseq platform and $2 \times 150$-bp paired-end reads were generated. The library preparation and sequencing were conducted by Novogene.

\section{Mapping to the reference genome and transcriptome assembly.}

The lncRNAseq raw data in FASTQ format was processed using Novogene's in-house perl scripts and the reads containing adaptors, unrecognized bases, and low-quality reads were removed. Based on the clean reads, percentage of the total number of bases where the Phred score is greater than 20 (Q20) and $30(\mathrm{Q} 30)$ was calculated. This indicated the base call accuracy and percentage of GC content in clean data. To align the reads, reference genome annotation files were downloaded from B. napus reference genome v5.0 (Chalhoub et al. 2014) and indexed using Bowtie v2.0.6 (Langmead and Salzberg 2012). The paired-end clean reads were aligned to this reference genome using TopHat v2.0.9 (Trapnell et al. 2012) and the mapped reads from each sample were assembled using the statistical segmentation model Scripture $\beta 2$ (Guttman et al. 2010) and Cufflinks v2.2.1 (Trapnell et al. 2010). A combination of these enabled us to distinguish the expressed loci from experimental noise. The spliced reads were then used to assemble the expressed segments and a probabilistic model to simultaneously assemble and provide a maximum-likelihood explanation of the expression at a specific locus. The program Scripture was run with the default parameters, while 'minfrags-per-transfrag $=0$ ' and '-library-type' were set for Cufflinks, with other parameters set to default. Sorting and removal of the duplicated reads and merging of the bam alignment file for each sample was accomplished by Picard- tools v1.41 and Samtools v.0.1.18.

\section{IncRNA detection and target prediction.}

The Cufflink assemblies generated from the samples were merged using the software Cuffmerge (Trapnell et al. 2010) to remove the replicates and artifacts. The remaining transcripts were subjected to the exon number filter (exon $\geq 2$ ) and transcript length filter $(>200 \mathrm{nt})$ to remove the other known noncoding RNA (rRNA, tRNA, snRNA, snoRNA, premiRNA, and pseudogenes) using Cuffcompare (Trapnell et al. 2010). Subsequently, the transcript sequences were classified and class-coded into candidate lincRNAs, intronic lncRNAs, and antisense lncRNAs. Four different software tools, including Coding Noncoding Index v2 (Sun et al. 2013); CPC 0.9-r2 (Kong et al. 2007), Pfam scan v1.3 (Punta et al. 2012), and phylogenic codon substitution frequency (Lin et al. 2011), were used to filter out the transcripts with coding potential. Only the transcripts without coding potential were considered as the candidate set of IncRNAs. The software program Cuffdiff v2.1.1 (Trapnell et al. 2010) was used to calculate the FPKMs in each sample. Fold changes for the DE lncRNAs were calculated as $\log _{2}$ (fold change $)=\log _{2}$ (FPKM susceptible/FPKM resistant). A $P$ value was assigned to each lncRNA and adjusted by the Benjamin and Hochberg approach (Benjamini and Hochberg 1995) for controlling the false-discovery rate. DE IncRNAs were considered significant when the absolute value of $\log _{2}$ (fold change) $>1$, with a $q$ value $<0.05$. The targets, where the lncRNA plays a cis role, were predicted by searching for genes at $100,000 \mathrm{bp}$ upstream and downstream of the IncRNAs. To predict the trans role, correlation between expression of lncRNAs and coding genes was calculated using the Pearson correlation coefficient, and genes having absolute correlation coefficient $>0.95$ were considered for further functional enrichment analysis.

\section{Classification and characterization of IncRNA functions.}

The lncRNAs acting as precursor of the known miRNAs were identified by BLASTn analysis (e-value $=1 \mathrm{e}-5)(\mathrm{J}$. Zhang et al. 2018). For this, all of the stem loop sequences of known B. napus miRNA were downloaded from the miRbase database and aligned with the IncRNA sequences showing significant differential expression. The hairpin loop formation in the lncRNAs was first checked using the miRNAFold server (Tav et al. 2016), and the secondary structure was plotted using the Vienna RNAfold web server (Gruber et al. 2008) and visualized using Forna (Kerpedjiev et al. 2015)

lncRNAs mimicking the endogenous miRNA targets were predicted by psRNA-Target V2.2017 (Dai et al. 2018) by subjecting them to stringent parameters of maximum expectation set to 3.5 and target accessibility set to 25.0. The interaction between the IncRNAs, miRNAs, and miRNA gene targets were visualized using Cytoscape v3.6.1 (Shannon et al. 2003)

NATs were subjected to RNAup (ViennaRNA Package v2.4.9) (Hofacker 2009; Lorenz et al. 2011) to calculate the thermodynamics of their interaction with their corresponding sense mRNAs and, therefore, determine the potential candidates of lncRNA-mRNA interaction. The potential dimerized secondary structures were calculated using RNAcofold (ViennaRNA Package v2.4.9) (Lorenz et al. 2011).

\section{qRT-PCR analysis.}

The lncRNAs responsive to the $P$. brassicae infection and their respective targets from $B$. napus were validated using qRT-PCR. This included the lncRNAs from chromosome A08, IncRNAs which are found to be regulating the targets on chromosome A08 and elsewhere in the genome, as well as some random IncRNAs which regulate target genes predicted to play a role in plant hormone signaling. Sequences of the primers used in qRT-PCR are listed in Supplementary Table S1. All qRT-PCR analyses were performed on QuantStudio 6 Flex Real-Time PCR System (Thermo Fisher Scientific) using PowerUP SYBR Green Master Mix from Applied Biosystems (Thermo Fisher Scientific). RNA isolated from roots of $\mathrm{R}$ and $\mathrm{S} \mathrm{DH}$ lines inoculated with $P$. brassicae were treated with RNase-free DNase I. The total 
RNA was reverse transcribed using random hexamers and the MiRNA-X miRNA First-Strand Synthesis Kit (Clontech Laboratories). Cycle threshold $(\mathrm{Ct})$ values were determined for each sample based on three biological replicates and two technical replicates from each of the biological replicate. Constitutively expressed gene UBC9 (Ubiquitin-conjugating enzyme 9) was used as an endogenous control. Comparison of gene expression was performed in the two samples, $\mathrm{R}$ and $\mathrm{S}$, each normalized using an endogenous control with $\mathrm{S}$ as the calibrator. Fold change was calculated using the formula $2^{-\Delta \Delta \mathrm{Ct}}=[(\mathrm{Ct}$ gene of interest $-\mathrm{Ct}$ endogenous control $) \mathrm{R}-(\mathrm{Ct}$ gene of interest $-\mathrm{Ct}$ endogenous control)S] (Schmittgen and Livak 2008).

\section{ACKNOWLEDGMENTS}

We thank G. Uhrig, S. Megha, and D. Mehta for their suggestions.

\section{AUTHOR-RECOMMENDED INTERNET RESOURCES}

psRNAtarget (version updated in 2017):

https://plantgrn.noble.org/psRNATarget/

B. napus reference genome v5.0:

http://www.genoscope.cns.fr/brassicanapus/data/

miRbase database: http://www.mirbase.org/

miRNAFold server https://evryrna.ibisc.univ-evry.fr/miRNAFold

Vienna RNAfold web server:

http://rna.tbi.univie.ac.at/cgi-bin/RNAWebSuite/RNAfold.cgi

Forna: http://rna.tbi.univie.ac.at/forna/

\section{LITERATURE CITED}

Ariel, F., Romero-Barrios, N., Jégu, T., Benhamed, M., and Crespi, M. 2015. Battles and hijacks: Noncoding transcription in plants. Trends Plant Sci. 20:362-371.

Asano, T., Kageyama, K., and Hyakumachi, M. 2000. Germination of surface-disinfected resting spores of Plasmodiophora brassicae and their root hair infection in turnip hairy roots. Mycoscience 41 : 49-54.

Benjamini, Y., and Hochberg, Y. 1995. Controlling the false discovery rate: A practical and powerful approach to multiple testing. J. R. Stat. Soc. Ser. B 57:289-300.

Burki, F., Kudryavtsev, A., Matz, M. V., Aglyamova, G. V., Bulman, S., Fiers, M., Keeling, P. J., and Pawlowski, J. 2010. Evolution of Rhizaria: New insights from phylogenomic analysis of uncultivated protists. BMC Evol. Biol. 10:377.

Cao, T., Srivastava, S., Rahman, M. H., Kav, N. N. V., Hotte, N., Deyholos, M. K., and Strelkov, S. E. 2008. Proteome-level changes in the roots of Brassica napus as a result of Plasmodiophora brassicae infection. Plant Sci. 174:97-115.

Caretto, S., Linsalata, V., Colella, G., Mita, G., and Lattanzio, V. 2015. Carbon fluxes between primary metabolism and phenolic pathway in plant tissues under stress. Int. J. Mol. Sci. 16:2637826394.

Chalhoub, B., Denoeud, F., Liu, S., Parkin, I. A., Tang, H., Wang, X., Chiquet, J., Belcram, H., Tong, C., Samans, B., Corréa, M., Da Silva, C., Just, J., Falentin, C., Koh, C. S., Le Clainche, I., Bernard, M., Bento, P., Noel, B., Labadie, K., Alberti, A., Charles, M., Arnaud, D., Guo, H., Daviaud, C., Alamery, S., Jabbari, K., Zhao, M., Edger, P. P., Chelaifa, H., Tack, D., Lassalle, G., Mestiri, I., Schnel, N., Le Paslier, M. C., Fan, G., Renault, V., Bayer, P. E., Golicz, A. A., Manoli, S., Lee, T. H., Thi, V. H., Chalabi, S., Hu, Q., Fan, C., Tollenaere, R., Lu, Y., Battail, C., Shen, J., Sidebottom, C. H., Wang, X., Canaguier, A., Chauveau, A., Bérard, A., Deniot, G., Guan, M., Liu, Z., Sun, F., Lim, Y. P., Lyons, E., Town, C. D., Bancroft, I., Wang, X., Meng, J., Ma, J., Pires, J. C., King, G. J., Brunel, D., Delourme, R., Renard, M., Aury, J. M., Adams, K. L., Batley, J., Snowdon, R. J., Tost, J., Edwards, D., Zhou, Y., Hua, W., Sharpe, A. G., Paterson, A. H., Guan, C., and Wincker, P. 2014. Early allopolyploid evolution in the post-Neolithic Brassica napus oilseed genome. Science 345:950-953.

Chauhan, S., Yogindran, S., and Rajam, M. V. 2017. Role of miRNAs in biotic stress reactions in plants. Indian J. Plant. Physiol. 22:514529.

Chen, J., Pang, W., Chen, B., Zhang, C., Piao, Z. 2016. Transcriptome analysis of Brassica rapa near-isogenic lines carrying clubroot-resistant and -susceptible alleles in response to Plasmodiophora brassicae during early infection. Front. Plant Sci. 6:1183.

Chen, J., Quan, M., and Zhang, D. 2015. Genome-wide identification of novel long non-coding RNAs in Populus tomentosa tension wood, opposite wood and normal wood xylem by RNA-seq. Planta 241 : $125-143$

Chen, X. 2009. Small RNAs and their roles in plant development. Annu. Rev. Cell Dev. Biol. 25:21-44.

Cui, J., Luan, Y., Jiang, N., Bao, H., and Meng, J. 2017. Comparative transcriptome analysis between resistant and susceptible tomato allows the identification of lncRNA16397 conferring resistance to Phytophthora infestans by co-expressing glutaredoxin. Plant J. 89:577-589.

Dai, X., Zhuang, Z., and Zhao, P. X. 2018. psRNATarget: A plant small RNA target analysis server (2017 release). Nucleic Acids Res. 46: W49-W54.

Devos, S., Laukens, K., Deckers, P., Van Der Straeten, D., Beeckman, T., Inzé, D., Van Onckelen, H., Witters, E., and Prinsen, E. 2006. A hormone and proteome approach to picturing the initial metabolic events during Plasmodiophora brassicae infection on Arabidopsis. Mol. PlantMicrobe Interact. 19:1431-1443.

Dixon, G. R. 2009. The occurrence and economic impact of Plasmodiophora brassicae and clubroot disease. J. Plant Growth Regul. 28: 194-202.

Dobson, R., Gabrielson, R., Baker, A., and Bennett, L. 1983. Effects of lime particle size and distribution and fertilizer formulation on clubroot disease caused by Plasmodiophora brassicae. Plant Dis. 67:50-52.

Ehness, R., Ecker, M., Godt, D. E., and Roitsch, T. 1997. Glucose and stress independently regulate source and sink metabolism and defense mechanisms via signal transduction pathways involving protein phosphorylation. Plant Cell 9:1825-1841.

Falcone Ferreyra, M. L., Rius, S. P., and Casati, P. 2012. Flavonoids: Biosynthesis, biological functions, and biotechnological applications. Front. Plant Sci. 3:222

Fei, W., Feng, J., Rong, S., Strelkov, S. E., Gao, Z., and Hwang, S.-F. 2016. Infection and gene expression of the clubroot pathogen Plasmodiophora brassicae in resistant and susceptible canola cultivars. Plant Dis. 100:824-828.

Gruber, A. R., Lorenz, R., Bernhart, S. H., Neuböck, R., and Hofacker, I. L. 2008. The Vienna RNA websuite. Nucleic Acids Res. 36:W70-W74.

Guttman, M., Amit, I., Garber, M., French, C., Lin, M. F., Feldser, D., Huarte, M., Zuk, O., Carey, B. W., Cassady, J. P., Cabili, M. N., Jaenisch, R., Mikkelsen, T. S., Jacks, T., Hacohen, N., Bernstein, B. E., Kellis, M., Regev, A., Rinn, J. L., and Lander, E. S. 2009. Chromatin signature reveals over a thousand highly conserved large non-coding RNAs in mammals. Nature 458:223-227.

Guttman, M., Garber, M., Levin, J. Z., Donaghey, J., Robinson, J., Adiconis, X., Fan, L., Koziol, M. J., Gnirke, A., Nusbaum, C., Rinn, J. L., Lander, E. S., and Regev, A. 2010. Ab initio reconstruction of cell type-specific transcriptomes in mouse reveals the conserved multi-exonic structure of lincRNAs. Nat. Biotechnol. 28:503-510.

Harakava, R. 2005. Genes encoding enzymes of the lignin biosynthesis pathway in Eucalyptus. Genet. Mol. Biol. 28:601-607.

Hasan, M. J., and Rahman, H. 2016. Genetics and molecular mapping of resistance to Plasmodiophora brassicae pathotypes 2, 3, 5, 6, and 8 in rutabaga (Brassica napus var napobrassica). Genome 59:805-815.

Hofacker, I. L. 2009. RNA secondary structure analysis using the Vienna RNA package. Curr. Protoc. Bioinf. 26:12.2.1-12.2.16.

Howard, R. J., Strelkov, S. E., and Harding, M. W. 2010. Clubroot of cruciferous crops-New perspectives on an old disease. Can. J. Plan Pathol. 32:43-57.

Hu, Q., Min, L., Yang, X., Jin, S., Zhang, L., Li, Y., Ma, Y., Qi, X., Li, D., Liu, H., Lindsey, K., Zhu, L., and Zhang, X. 2018. Laccase GHlac1 modulates broad-spectrum biotic stress tolerance via manipulating phenylpropanoid pathway and jasmonic acid synthesis. Plant Physiol. 176:1808-1823.

Huang, B., and Zhang, R. 2014. Regulatory non-coding RNAs: Revolutionizing the RNA world. Mol. Biol. Rep. 41:3915-3923.

Huang, L., Dong, H., Zhou, D., Li, M., Liu, Y., Zhang, F., Feng, Y., Yu, D., Lin, S., and Cao, J. 2018. Systematic identification of long non-coding RNAs during pollen development and fertilization in Brassica rapa. Plant J. 96:203-222.

Hwang, S.-F., Strelkov, S. E., Feng, J., Gossen, B. D., and Howard, R. J. 2012. Plasmodiophora brassicae: A review of an emerging pathogen of the Canadian canola (Brassica napus) crop. Mol. Plant Pathol. 13: 105-113.

Ietswaart, R., Rosa, S., Wu, Z., Dean, C., and Howard, M. 2017. Cell-sizedependent transcription of $F L C$ and its antisense long non-coding RNA COOLAIR explain cell-to-cell expression variation. Cell Syst. 4: 622-635.e9. 
Jabnoune, M., Secco, D., Lecampion, C., Robaglia, C., Shu, Q., and Poirier, Y. 2013. A rice cis-natural antisense RNA acts as a translational enhancer for its cognate mRNA and contributes to phosphate homeostasis and plant fitness. Plant Cell 25:4166-4182.

Joshi, R. K., Megha, S., Basu, U., Rahman, M. H., and Kav, N. N. 2016 Genome wide identification and functional prediction of long noncoding RNAs responsive to Sclerotinia sclerotiorum infection in Brassica napus. PLoS One 11:e0158784.

Kang, C., and Liu, Z. 2015. Global identification and analysis of long noncoding RNAs in diploid strawberry Fragaria vesca during flower and fruit development. BMC Genomics 16:815.

Kapranov, P., Cheng, J., Dike, S., Nix, D. A., Duttagupta, R., Willingham, A. T., Stadler, P. F., Hertel, J., Hackermüller, J., Hofacker, I. L., Bell, I. Cheung, E., Drenkow, J., Dumais, E., Patel, S., Helt, G., Ganesh, M., Ghosh, S., Piccolboni, A., Sementchenko, V., Tammana, H., and Gingeras, T. R. 2007. RNA maps reveal new RNA classes and a possible function for pervasive transcription. Science 316:1484-1488.

Kerpedjiev, P., Hammer, S., and Hofacker, I. L. 2015. Forna (force-directed RNA): Simple and effective online RNA secondary structure diagrams. Bioinformatics 31:3377-3379.

Kong, L., Zhang, Y., Ye, Z.-Q., Liu, X.-Q., Zhao, S.-Q., Wei, L., and Gao, G. 2007. CPC: Assess the protein-coding potential of transcripts using sequence features and support vector machine. Nucleic Acids Res. 35: W345-W349.

Kopp, F., and Mendell, J. T. 2018. Functional classification and experimental dissection of long noncoding RNAs. Cell 172:393-407.

Kornienko, A. E., Guenzl, P. M., Barlow, D. P., and Pauler, F. M. 2013. Gene regulation by the act of long non-coding RNA transcription. BMC Biol. 11:59.

Koroban, N. V., Kudryavtseva, A. V., Krasnov, G. S., Sadritdinova, A. F., Fedorova, M. S., Snezhkina, A. V., Bolsheva, N. L., Muravenko, O. V. Dmitriev, A. A., and Melnikova, N. V. 2016. The role of microRNA in abiotic stress response in plants. Mol. Biol. 50:337-343.

Kowata-Dresch, L., and May-De Mio, L. 2012. Clubroot management of highly infested soils. Crop Prot. 35:47-52.

Kumar, R. 2014. Role of microRNAs in biotic and abiotic stress responses in crop plants. Appl. Biochem. Biotechnol. 174:93-115.

Kutter, C., Schöb, H., Stadler, M., Meins, F., Jr., and Si-Ammour, A. 2007. MicroRNA-mediated regulation of stomatal development in Arabidopsis. Plant Cell 19:2417-2429.

Langmead, B., and Salzberg, S. L. 2012. Fast gapped-read alignment with Bowtie 2. Nat. Methods 9:357-359.

Le Roy, J., Huss, B., Creach, A., Hawkins, S., and Neutelings, G. 2016 Glycosylation is a major regulator of phenylpropanoid availability and biological activity in plants. Front. Plant Sci. 7:735.

Li, L., Eichten, S. R., Shimizu, R., Petsch, K., Yeh, C. T., Wu, W., Chettoor, A. M., Givan, S. A., Cole, R. A., Fowler, J. E., Evans, M. M., Scanlon, M. J., Yu, J., Schnable, P. S., Timmermans, M. C., Springer, N. M., and Muehlbauer, G. J. 2014. Genome-wide discovery and characterization of maize long non-coding RNAs. Genome Biol. 15:R40.

Lin, M. F., Jungreis, I., and Kellis, M. 2011. PhyloCSF: A comparative genomics method to distinguish protein coding and non-coding regions. Bioinformatics 27:i275-i282.

Liu, J., Jung, C., Xu, J., Wang, H., Deng, S., Bernad, L., Arenas-Huertero, C., and Chua, N. H. 2012. Genome-wide analysis uncovers regulation of long intergenic noncoding RNAs in Arabidopsis. Plant Cell 24: 4333-4345.

Liu, X., Li, D., Zhang, D., Yin, D., Zhao, Y., Ji, C., Zhao, X., Li, X., He, Q. Chen, R., Hu, S., and Zhu, L. 2018. A novel antisense long noncoding RNA, TWISTED LEAF, maintains leaf blade flattening by regulating its associated sense R2R3-MYB gene in rice. New Phytol. 218:774-788.

Lorenz, R., Bernhart, S. H., Höner Zu Siederdissen, C., Tafer, H., Flamm, C., Stadler, P. F., and Hofacker, I. L. 2011. ViennaRNA Package 2.0. Algorithms Mol. Biol. 6:26.

Luan, M., Xu, M., Lu, Y., Zhang, Q., Zhang, L., Zhang, C., Fan, Y., Lang, Z., and Wang, L. 2014. Family-wide survey of miR169s and NF-YAs and their expression profiles response to abiotic stress in maize roots. PLoS One 9:e91369.

Macfarlane, I. 1970. Germination of resting spores of Plasmodiophora brassicae. Trans. Br. Mycol. Soc. 55:97-112.

Mattick, J. S., and Rinn, J. L. 2015. Discovery and annotation of long noncoding RNAs. Nat. Struct. Mol. Biol. 22:5-7.

Meng, Y., Shao, C., Wang, H., and Jin, Y. 2012. Target mimics: An embedded layer of microRNA-involved gene regulatory networks in plants. BMC Genomics 13:197.

Morris, K. V., and Mattick, J. S. 2014. The rise of regulatory RNA. Nat. Rev. Genet. 15:423-437.
Nejat, N., and Mantri, N. 2018. Emerging roles of long non-coding RNAs in plant response to biotic and abiotic stresses. Crit. Rev. Biotechnol. 38:93-105.

Peng, G., Lahlali, R., Hwang, S.-F., Pageau, D., Hynes, R. K., McDonald, M. R., Gossen, B. D., and Strelkov, S. E. 2014. Crop rotation, cultivar resistance, and fungicides/biofungicides for managing clubroot (Plasmodiophora brassicae) on canola. Can. J. Plant Pathol. 36:99-112.

Piao, Z. Y., Deng, Y. Q., Choi, S. R., Park, Y. J., and Lim, Y. P. 2004. SCAR and CAPS mapping of $C R b$, a gene conferring resistance to Plasmodiophora brassicae in Chinese cabbage ( Brassica rapa ssp. pekinensis). Theor. Appl. Genet. 108:1458-1465.

Punta, M., Coggill, P. C., Eberhardt, R. Y., Mistry, J., Tate, J., Boursnell, C., Pang, N., Forslund, K., Ceric, G., Clements, J., Heger, A., Holm, L., Sonnhammer, E. L., Eddy, S. R., Bateman, A., and Finn, R. D. 2012. The Pfam protein families database. Nucleic Acids Res. 40:D290-D301.

Rahman, H. 2016. UA AlfaGold Clearfield herbicide-tolerant spring Brassica napus canola developed from winter $\times$ spring canola cross. Can. J. Plant Sci. 97:144-146.

Rahman, H., Peng, G., Yu, F., Falk, K. C., Kulkarni, M., and Selvaraj, G. 2014. Genetics and breeding for clubroot resistance in Canadian spring canola (Brassica napus L.). Can. J. Plant Pathol. 36:122-134.

Rai, M. I., Alam, M., Lightfoot, D. A., Gurha, P., and Afzal, A. J. Classification and experimental identification of plant long non-coding RNAs. Genomics In press. doi.org/10.1016/j.ygeno.2018.04.014

Rojas, C. M., Senthil-Kumar, M., Tzin, V., and Mysore, K. S. 2014. Regulation of primary plant metabolism during plant-pathogen interactions and its contribution to plant defense. Front. Plant Sci. 5:17.

Schmittgen, T. D., and Livak, K. J. 2008. Analyzing real-time PCR data by the comparative C(T) method. Nat. Protoc. 3:1101-1108.

Seo, J. S., Sun, H. X., Park, B. S., Huang, C. H., Yeh, S. D., Jung, C., and Chua, N. H. 2017. ELF18-INDUCED LONG-NONCODING RNA associates with mediator to enhance expression of innate immune response genes in Arabidopsis. Plant Cell 29:1024-1038.

Shannon, P., Markiel, A., Ozier, O., Baliga, N. S., Wang, J. T., Ramage, D., Amin, N., Schwikowski, B., and Ideker, T. 2003. Cytoscape: A software environment for integrated models of biomolecular interaction networks. Genome Res. 13:2498-2504.

Shumayla, S., Sharma, S., Taneja, M., Tyagi, S., Singh, K., and Upadhyay, S. K. 2017. Survey of high throughput RNA-seq data reveals potential roles for lncRNAs during development and stress response in bread wheat. Front. Plant Sci. 8:1019.

Song, J. H., Cao, J. S., and Wang, C. G. 2013. BcMF11, a novel non-coding RNA gene from Brassica campestris, is required for pollen development and male fertility. Plant Cell Rep. 32:21-30.

Sun, L., Luo, H., Bu, D., Zhao, G., Yu, K., Zhang, C., Liu, Y., Chen, R., and Zhao, Y. 2013. Utilizing sequence intrinsic composition to classify proteincoding and long non-coding transcripts. Nucleic Acids Res. 41:e166.

Tav, C., Tempel, S., Poligny, L., and Tahi, F. 2016. miRNAFold: A web server for fast miRNA precursor prediction in genomes. Nucleic Acids Res. 44:W181-W184.

Tayeh, C., Randoux, B., Vincent, D., Bourdon, N., and Reignault, P. 2014 Exogenous trehalose induces defenses in wheat before and during a biotic stress caused by powdery mildew. Phytopathology 104:293-305.

Tewari, J. P., Strelkov, S. E., Orchard, D., Hartman, M., Lange, R. M., and Turkington, T. K. 2005. Identification of clubroot of crucifers on canola (Brassica napus) in Alberta. Can. J. Plant Pathol. 27:143-144.

Trapnell, C., Roberts, A., Goff, L., Pertea, G., Kim, D., Kelley, D. R., Pimentel, H., Salzberg, S. L., Rinn, J. L., and Pachter, L. 2012. Differential gene and transcript expression analysis of RNA-sec experiments with TopHat and Cufflinks. Nat. Protoc. 7:562-578.

Trapnell, C., Williams, B. A., Pertea, G., Mortazavi, A., Kwan, G., van Baren, M. J., Salzberg, S. L., Wold, B. J., and Pachter, L. 2010. Transcript assembly and quantification by RNA-Seq reveals unannotated transcripts and isoform switching during cell differentiation. Nat. Biotechnol. 28:511-515.

Treutter, D. 2006. Significance of flavonoids in plant resistance: A review. Environ. Chem. Lett. 4:147-157.

Verma, S. S., Rahman, M. H., Deyholos, M. K., Basu, U., and Kav, N. N. 2014. Differential expression of miRNAs in Brassica napus roo following infection with Plasmodiophora brassicae. PLoS One 9: e86648.

Vogt, T. 2010. Phenylpropanoid biosynthesis. Mol. Plant 3:2-20.

Voorrips, R. E., and Visser, D. L. 1993. Examination of resistance to clubroot in accessions of Brassica oleracea using a glasshouse seedling test. Neth. J. Plant Pathol. 99:269-276.

Wallenhammar, A. C. 1996. Prevalence of Plasmodiophora brassicae in a spring oilseed rape growing area in central Sweden and factors influencing soil infestation levels. Plant Pathol. 45:710-719. 
Wang, C. Y., Liu, S. R., Zhang, X. Y., Ma, Y. J., Hu, C. G., and Zhang, J. Z. 2017. Genome-wide screening and characterization of long non-coding RNAs involved in flowering development of trifoliate orange (Poncirus trifoliata L. Raf.). Sci. Rep. 7:43226.

Wang, H., Chung, P. J., Liu, J., Jang, I. C., Kean, M. J., Xu, J., and Chua, N. H. 2014. Genome-wide identification of long noncoding natural antisense transcripts and their responses to light in Arabidopsis. Genome Res. 24:444-453.

Wang, J., Yu, W., Yang, Y., Li, X., Chen, T., Liu, T., Ma, N., Yang, X., Liu, R., and Zhang, B. 2015. Genome-wide analysis of tomato long noncoding RNAs and identification as endogenous target mimic for microRNA in response to TYLCV infection. Sci. Rep. 5:16946.

Wang, N., Cao, P., Xia, W., Fang, L., and Yu, H. 2017. Identification and characterization of long non-coding RNAs in response to early infection by Melampsora larici-populina using genome-wide high-throughput RNA sequencing. Tree Genet. Genomes 13:34.

Wight, M., and Werner, A. 2013. The functions of natural antisense transcripts. Essays Biochem. 54:91-101.

Williams, P. H. 1966. A system for the determination of races of Plasmodiophora brassicae that infect cabbage and rutabaga. Phytopathology 56:624-626.

Wilusz, J. E., Sunwoo, H., and Spector, D. L. 2009. Long noncoding RNAs: Functional surprises from the RNA world. Genes Dev. 23:1494-1504.

Wu, H., Yang, L., and Chen, L.-L. 2017. The diversity of long noncoding RNAs and their generation. Trends Genet. 33:540-552.

Wu, H.-J., Wang, Z.-M., Wang, M., and Wang, X.-J. 2013. Wide-spread long non-coding RNAs as endogenous target mimics for microRNAs in plants. Plant Physiol. 161:1875-1884.

Xiao, W., Sheen, J., and Jang, J. C. 2000. The role of hexokinase in plant sugar signal transduction and growth and development. Plant Mol. Biol. 44:451-461.

Xin, M., Wang, Y., Yao, Y., Song, N., Hu, Z., Qin, D., Xie, C., Peng, H., Ni, Z., and Sun, Q. 2011. Identification and characterization of wheat long non-protein coding RNAs responsive to powdery mildew infection and heat stress by using microarray analysis and SBS sequencing. BMC Plant Biol. 11:61.
Yeung, E. C., and Saxena, P. K. 2005. Histological techniques. Pages 517-537 in: Protocol for Somatic Embryogenesis in Woody Plants. S. M. Jain and P. K. Gupta, eds. Springer, Dordrecht, The Netherlands.

Zaynab, M., Fatima, M., Abbas, S., Umair, M., Sharif, Y., and Raza, M. A. 2018. Long non-coding RNAs as molecular players in plant defense against pathogens. Microb. Pathog. 121:277-282.

Zhang, J., Wei, L., Jiang, J., Mason, A. S., Li, H., Cui, C., Chai, L., Zheng, B., Zhu, Y., Xia, Q., Jiang, L., and Fu, D. 2018. Genome-wide identification, putative functionality and interactions between lncRNAs and miRNAs in Brassica species. Sci. Rep. 8: Article 4960.

Zhang, L., Wang, M., Li, N., Wang, H., Qiu, P., Pei, L., Xu, Z., Wang, T., Gao, E., Liu, J., Liu, S., Hu, Q., Miao, Y., Lindsey, K., Tu, L., Zhu, L., and Zhang, X. 2018. Long noncoding RNAs involve in resistance to Verticillium dahliae, a fungal disease in cotton. Plant Biotechnol. J. 16: 1172-1185.

Zhang, X., Liu, Y., Fang, Z., Li, Z., Yang, L., Zhuang, M., Zhang, Y., and Lv, H. 2016. Comparative transcriptome analysis between broccoli (Brassica oleracea var. italica) and wild cabbage (Brassica macrocarpa Guss.) in response to Plasmodiophora brassicae during different infection stages. Front. Plant Sci. 7:1929.

Zhang, X., Zou, Z., Gong, P., Zhang, J., Ziaf, K., Li, H., Xiao, F., and Ye, Z. 2011. Over-expression of microRNA169 confers enhanced drought tolerance to tomato. Biotechnol. Lett. 33:403-409.

Zhao, J.-P., Jiang, X.-L., Zhang, B.-Y., and Su, X.-H. 2012. Involvement of microRNA-mediated gene expression regulation in the pathological development of stem canker disease in Populus trichocarpa. PLoS One 7:e44968.

Zhu, Q. H., Stephen, S., Taylor, J., Helliwell, C. A., and Wang, M. B. 2014. Long noncoding RNAs responsive to Fusarium oxysporum infection in Arabidopsis thaliana. New Phytol. 201:574-584.

Zubko, E., and Meyer, P. 2007. A natural antisense transcript of the Petunia hybrida Sho gene suggests a role for an antisense mechanism in cytokinin regulation. Plant J. 52:1131-1139. 